\title{
Extreme points of the core of possibility measures and maxitive $p$-boxes
}

\author{
IGNACIO MONTES \\ Department of Statistics and O.R., University of Oviedo, C/Federico García Lorca, 18 \\ Oviedo, 33007 Spain \\ imontes@uniovi.es \\ ENRIQUE MIRANDA \\ Department of Statistics and O.R., University of Oviedo, C/Federico García Lorca, 18 \\ Oviedo, 33007 Spain \\ mirandaenrique@uniovi.es \\ Received (received date) \\ Revised (revised date)
}

\begin{abstract}
Under an epistemic interpretation, an upper probability can be regarded as equivalent to the set of probability measures it dominates, sometimes referred to as its core. In this paper, we study the properties of the number of extreme points of the core of a possibility measure, and investigate in detail those associated with (uni- and bi-)variate $p$-boxes, that model the imprecise information about a cumulative distribution function.

Keywords: Belief functions, possibility measures, probability boxes, credal sets, extreme points.
\end{abstract}

\section{Introduction}

Capacities, or non-additive measures ${ }^{2}$, are mathematical models that serve as an alternative to probability theory. They are of particular interest in situations of ambiguous or imprecise knowledge, and can thus be embedded within the theory of imprecise probabilities. As particular cases, they include possibility measures ${ }^{7}$, belief functions ${ }^{23}, n$-monotone capacities ${ }^{2}$ or coherent lower and upper probabilities ${ }^{27}$, amongst others.

In this paper, we take a non-additive measure $\mu$ defined on the power set of a finite space $\mathcal{X}$, and consider its core or credal set $\mathcal{M}(\mu)$, which is the set of probability measures dominated by $\mu$ on all subsets of $\mathcal{X}$. This set is useful for instance if we give $\mu$ an epistemic interpretation as a model for the imprecise knowledge of some probability measure $P$, in the sense that all we can give about $P(A)$ is an upper bound $\mu(A)$, and this for every set $A$; but it has also arisen in other contexts, such as random set theory ${ }^{4}$ or game theory ${ }^{5,24}$.

We focus here on coherent upper probabilities $\mu^{27}$, which are those whose associated credal set $\mathcal{M}(\mu)$ is non-empty and such that $\mu$ is the upper envelope of 
$\mathcal{M}(\mu)$. These include as particular cases most of the existing models of non-additive measures in the literature. In those cases, the set $\mathcal{M}(\mu)$, and as a consequence the non-additive measure $\mu$, is determined by its vertices or extreme points; these can then be used for a number of practical purposes, for instance when considering graphical models with sets of probabilities ${ }^{3}$, updating the probabilistic model under new information ${ }^{8}$, or giving the solution of a game whose information is modeled by $\mu^{5,24}$.

It was proven by Wallner ${ }^{28}$ that the credal set associated with a coherent upper probability $\mu$ has at most $n$ ! different extreme points, where $n$ is the cardinality of the possibility space. This bound was the same that Dempster established earlier for the particular case of plausibility functions ${ }^{4}$ and Shapley for 2-alternating capacities ${ }^{24}$. On the other hand, in the case of possibility measures, that constitute a particular case of plausibility functions, the maximum number of extreme points reduces to $2^{n-1} 12,14$.

In this paper, we deepen into this study by considering the formula established in ${ }^{22}$ for the number of extreme points of the core of a possibility measure, in terms of the cardinalities of its focal elements. In particular, we characterize in which cases the maximal number of extreme points is attained. Then we consider another particular model of non-additive measures: probability boxes ${ }^{9}$, or $p$-boxes for short, that may be regarded as imprecise distribution functions. In the univariate case, $p$-boxes are related to plausibility functions ${ }^{25}$, and a study of the number of extreme points of their core was recently made in ${ }^{16}$. The situation is more involved in the bivariate case, because there a $p$-box need not be equivalent to a coherent upper probability, let alone a plausibility function ${ }^{21}$. Nevertheless, in ${ }^{17}$ necessary and sufficient conditions for a bivariate $p$-box to be equivalent to a possibility measure were established. We use those conditions here to give the tightest possible bound on the number of extreme points of the core of a maxitive bivariate $p$-box, in terms of the cardinalities of the possibility spaces where it is defined. Moreover, we characterize in which cases this bound is attained.

The remainder of this paper is organized as follows: in Section 2 we recall the existing characterization of the extreme points of the core of a plausibility function; in Section 3 we consider the particular case of possibility measures, and study when the maximal number of extreme points are attained. In Section 4 we focus on those $p$-boxes that are associated with a possibility measure, both in the univariate and in the bivariate case. Some additional comments and remarks are given in Section 5. In order to ease the reading, the proofs of our results as well as some auxiliary lemmas have been gathered in an Appendix.

\section{Extreme points for plausibility functions}

\subsection{Basic definitions}

The main model we shall consider in this paper are belief and plausibility functions, that play a key role in the theory of evidence developed by Shafer ${ }^{23}$. Let $\mathcal{X}=$ 
$\left\{x_{1}, \ldots, x_{n}\right\}$ be a finite possibility space.

Definition 1. A basic probability assignment is a function $m: \mathcal{P}(\mathcal{X}) \rightarrow[0,1]$ such that $m(\emptyset)=0$ and $\sum_{A \subseteq \mathcal{X}} m(A)=1$. Its associated belief Bel and plausibility $\mathrm{Pl}$ functions are given by:

$$
\operatorname{Bel}(A)=\sum_{B \subseteq A} m(B) \text { and } \operatorname{Pl}(A)=\sum_{B \cap A \neq \emptyset} m(B) .
$$

Bel and $\mathrm{Pl}$ are conjugate functions because they satisfy the relation $\operatorname{Bel}(A)=1-$ $\operatorname{Pl}\left(A^{c}\right)$, and moreover $\operatorname{Bel}(A) \leq \operatorname{Pl}(A)$ for any $A \subseteq \mathcal{X}$. The subsets $E$ of $\Omega$ such that $m(E)>0$ are called the focal sets of $m$, and can be used to determine the associated belief and plausibility function by means of Equation (1).

One interpretation of belief and plausibility functions is that of envelopes of a family of probability measures. This family is also called their core or credal set:

$$
\mathcal{M}(\mathrm{Pl})=\{P \text { prob. } \mid \operatorname{Bel}(A) \leq P(A) \leq \operatorname{Pl}(A) \forall A \subseteq \mathcal{X}\} .
$$

The core allows us to link evidence theory with imprecise probability theory ${ }^{27} . \mathrm{Bel}$ and $P l$ are the lower and upper envelopes of $\mathcal{M}(\mathrm{Pl})$, respectively:

$\operatorname{Bel}(A)=\min \{P(A): P \in \mathcal{M}(\mathrm{Pl})\}$ and $\operatorname{Pl}(A)=\max \{P(A): P \in \mathcal{M}(\mathrm{Pl})\} \forall A \subseteq \mathcal{X}$.

The set $\mathcal{M}(\mathrm{Pl})$ is a closed and convex subset of the finite-dimensional space of probability measures on $\mathcal{P}(\mathcal{X})$, and it is therefore characterized by its extreme points. We say that $P \in \mathcal{M}(\mathrm{Pl})$ is an extreme point when there are not $P_{1}, P_{2} \in \mathcal{M}(\mathrm{Pl})$ with $P_{1} \neq P_{2}$ and $\alpha \in(0,1)$ such that $P=\alpha P_{1}+(1-\alpha) P_{2}$. Then if $\left\{P_{1}, \ldots, P_{m}\right\}$ are the extreme points of $\mathcal{M}(\mathrm{Pl})$, it follows that for every $P \in \mathcal{M}(\mathrm{Pl})$ there are $\alpha_{1}, \ldots, \alpha_{m}$ such that $\alpha_{i} \geq 0$ for any $i=1, \ldots, m, \alpha_{1}+\ldots+\alpha_{m}=1$ and $P=\alpha_{1} \cdot P_{1}+\ldots+\alpha_{m} \cdot P_{m}$.

\subsection{Extreme points of the core of a plausibility function}

It is well-known ${ }^{1,4,24}$ that there is a connection between the extreme points of $\mathcal{M}(\mathrm{Pl})$ and the permutations of $\{1, \ldots, n\}$ : any permutation $\sigma$ of $\{1, \ldots, n\}$ determines an extreme point of $\mathcal{M}(\mathrm{Pl})$ by means of:

$$
\begin{aligned}
& P_{\sigma}\left(\left\{x_{\sigma(1)}\right\}\right)=\operatorname{Pl}\left(\left\{x_{1}\right\}\right), \\
& P_{\sigma}\left(\left\{x_{\sigma(i)}\right\}\right)=\operatorname{Pl}\left(\left\{x_{\sigma(1)}, \ldots, x_{\sigma(i)}\right\}\right)-\operatorname{Pl}\left(\left\{x_{\sigma(1)}, \ldots, x_{\sigma(i-1)}\right\}\right), \forall i=2, \ldots, n,
\end{aligned}
$$

and, conversely, any extreme point of $\mathcal{M}(P l)$ is associated with a permutation $\sigma$ in the manner described above. Hence, the maximal number of extreme points of $\mathcal{M}(\mathrm{Pl})$ is $n$ !, where $n$ is the cardinality of the possibility space. However, this number is not always attained, because different permutations may give rise to the same extreme point by means of Equation (2).

The extreme points of the credal set associated with a plausibility function were recently investigated in ${ }^{16}$, and a new representation of the extreme points in terms of counting vectors was introduced. This representation helped establishing a number of properties of the number of extreme points of $\mathcal{M}(\mathrm{Pl})$. In particular, it 
was established ${ }^{16}$ that $\mathcal{M}(\mathrm{Pl})$ has $n$ ! extreme points if and only if all the sets of cardinality two are focal.

\section{Extreme points for possibility measures}

We focus next on a particular case of plausibility and belief functions: possibility and necessity measures. A possibility measure ${ }^{7,29} \Pi: \mathcal{P}(\mathcal{X}) \rightarrow[0,1]$ is a supremumpreserving function: $\Pi(A)=\sup _{x \in A} \Pi(\{x\}) \forall A \subseteq \mathcal{X}$. Its conjugate function is called a necessity measure $N(A)=1-\Pi\left(A^{c}\right)$. In the context of this paper, where $\mathcal{X}$ is finite, they are equivalent to maxitive and minitive measures, because they satisfy

$$
\Pi(A \cup B)=\max \{\Pi(A), \Pi(B)\} \text { and } N(A \cap B)=\min \{N(A), N(B)\} \forall A, B \subseteq \mathcal{X} .
$$

Possibility and necessity measures correspond to the particular case of plausbility and belief functions whose focal elements $E_{1}, \ldots, E_{l}$ are nested, in the sense that $E_{1} \subset \ldots \subset E_{l}$, where $\subset$ is used to denote strict inclusion. As a consequence, we can compute the extreme points of the core $\mathcal{M}(\Pi)$ of a possibility measure by means of Equation (2). We denote by $|\operatorname{ext}(\mathcal{M}(\Pi))|$ the number of extreme points on $\mathcal{M}(\Pi)$.

Consider thus a possibility measure $\Pi: \mathcal{P}(\mathcal{X}) \rightarrow[0,1]$ on $\mathcal{X}=\left\{x_{1}, \ldots, x_{n}\right\}$, and let $E_{1} \subset \ldots \subset E_{l}$ be its focal sets. We shall assume that $E_{l}=\mathcal{X}$, which means that any singleton has a positive possibility measure; it is easy to extend the results to the general case, simply by removing the elements in $\mathcal{X} \backslash E_{l}$.

Let us define

$$
k_{1}=\left|E_{1}\right| \text { and } k_{i}=\left|E_{i} \backslash E_{i-1}\right| \forall i=2, \ldots, l .
$$

Since $\left\{E_{1}, E_{2} \backslash E_{1}, \ldots, E_{l} \backslash E_{l-1}\right\}$ is a partition of $\mathcal{X}$, we deduce that $k_{1}+\ldots+k_{l}=n$.

The following theorem gives the exact number of extreme points of the credal set associated with a possibility measure in terms of the cardinalities of the focal sets. It improves upon earlier results by Kroupa $\left({ }^{11},{ }^{12}\right)$, where only a lower and an upper bound of the number of extreme points are given.

Theorem 1. ${ }^{22}$ Let $\Pi: \mathcal{P}(\mathcal{X}) \rightarrow[0,1]$ be a possibility measure with focal sets $E_{1} \subset \ldots \subset E_{l}=\mathcal{X}$. The number of extreme points of $\mathcal{M}(\Pi)$ is

$$
k_{1}\left(1+k_{2}\right) \cdot \ldots \cdot\left(1+k_{l}\right),
$$

where $k_{1}, \ldots, k_{l}$ are given by Equation (3).

Thus, the number of extreme points only depends on the cardinalities of the focal sets but not on their masses. For this reason, from now on when considering possibility measures we shall omit the values of their mass function and restrict our attention to the focal sets.

We shall use extensively the formula given in Theorem 1 to investigate some properties of the number of extreme points induced by a possibility measure. In particular, we will also characterize the conditions a possibility measure must satisfy in order for this number to be maximal. 
Proposition 1. Let $\Pi: \mathcal{P}(\mathcal{X}) \rightarrow[0,1]$ be a possibility measure with focal sets $E_{1} \subset \ldots \subset E_{l}=\mathcal{X}$. Assume that there exists a set $E^{*}$ such that $E_{i} \subset E^{*} \subset E_{i+1}$ for some $i=1, \ldots, l-1$, and consider a possibility measure $\Pi^{\prime}: \mathcal{P}(\mathcal{X}) \rightarrow[0,1]$ with focal sets $E_{1} \subset \ldots \subset E_{i} \subset E^{*} \subset E_{i+1} \subset \ldots \subset E_{l}$. Then, $\mathcal{M}\left(\Pi^{\prime}\right)$ has more extreme points than $\mathcal{M}(\Pi)$.

With a similar reasoning we obtain the following:

Proposition 2. Let $\Pi: \mathcal{P}(\mathcal{X}) \rightarrow[0,1]$ be a possibility measure with focal sets $E_{1} \subset$ $\ldots \subset E_{l}=\mathcal{X}$. Assume that there exists a non-empty set $E^{*}$ such that $E^{*} \subset E_{1}$, and consider a possibility measure $\Pi^{\prime}: \mathcal{P}(\mathcal{X}) \rightarrow[0,1]$ with focal sets $E^{*} \subset E_{1} \subset \ldots \subset E_{l}$. Then,

(i) If $\left|E^{*}\right|>1, \mathcal{M}\left(\Pi^{\prime}\right)$ has more extreme points than $\mathcal{M}(\Pi)$.

(ii) If $\left|E^{*}\right|=1, \mathcal{M}\left(\Pi^{\prime}\right)$ and $\mathcal{M}(\Pi)$ have the same number of extreme points.

The underlying idea to these two results is that the focal sets of a possibility measure $\Pi$ induce the partition $\left\{E_{1}, E_{2} \backslash E_{1}, \ldots, E_{l} \backslash E_{l-1}\right\}$ of the possibility space, and that if the partition induced by $\Pi^{\prime}$ is finer than the one induced by $\Pi$, then $\mathcal{M}\left(\Pi^{\prime}\right)$ has at least as many extreme points as $\mathcal{M}(\Pi)$.

It was established in ${ }^{14}$ that the maximal number of extreme points on $\mathcal{M}(\Pi)$ is $2^{n-1}$, where $n=|\mathcal{X}|$ (this is also a consequence of Theorem 1). Our previous results allow us to determine the possibility measures where this maximal number is attained.

Corollary 1. Given a possibility measure $\Pi: \mathcal{P}(\mathcal{X}) \rightarrow[0,1]$ with focal sets $E_{1} \subset$ $\ldots \subset E_{l}$, its core $\mathcal{M}(\Pi)$ has $2^{n-1}$ extreme points if and only if one of the following statements holds:

(i) $l=n$ and $\left|E_{i}\right|=i$ for any $i=1, \ldots, n$.

(ii) $l=n-1,\left|E_{1}\right|=2$ and $\left|E_{i}\right|=i+1$ for any $i=2, \ldots, n-1$.

We conclude this section with a recursive algorithm that computes the extreme points of the credal set associated with a possibility measure. Some interesting related results under a geometric approach can be found in ${ }^{22}$. Consider a possibility measure $\Pi$ with focal sets $E_{1} \subset \ldots \subset E_{l}$, and let $k_{1}, \ldots, k_{l}$ be given by Equation (3). In order to simplify the notation, we shall assume without loss of generality that for any $x_{r} \in E_{j}$ and $x_{s} \in E_{j+1} \backslash E_{j}$ it holds that $r<s$ (i.e., the elements of $\mathcal{X}$ follow the same order as the focal sets). For any $P \in \mathcal{M}(\Pi)$, denote by $x_{P}^{*}$ the element:

$$
x_{P}^{*}=\max \left\{x_{i}: P\left(\left\{x_{i}\right\}\right)>0\right\} .
$$

Finally, let $\delta_{x}$ denote the degenerate probability on $x \in \mathcal{X}$, determined by the mass function $\delta_{x}(\{x\})=1$. The procedure for computing the extreme points of $\mathcal{M}(\Pi)$ is described in Algorithm 1.

The following example illustrates the algorithm. 


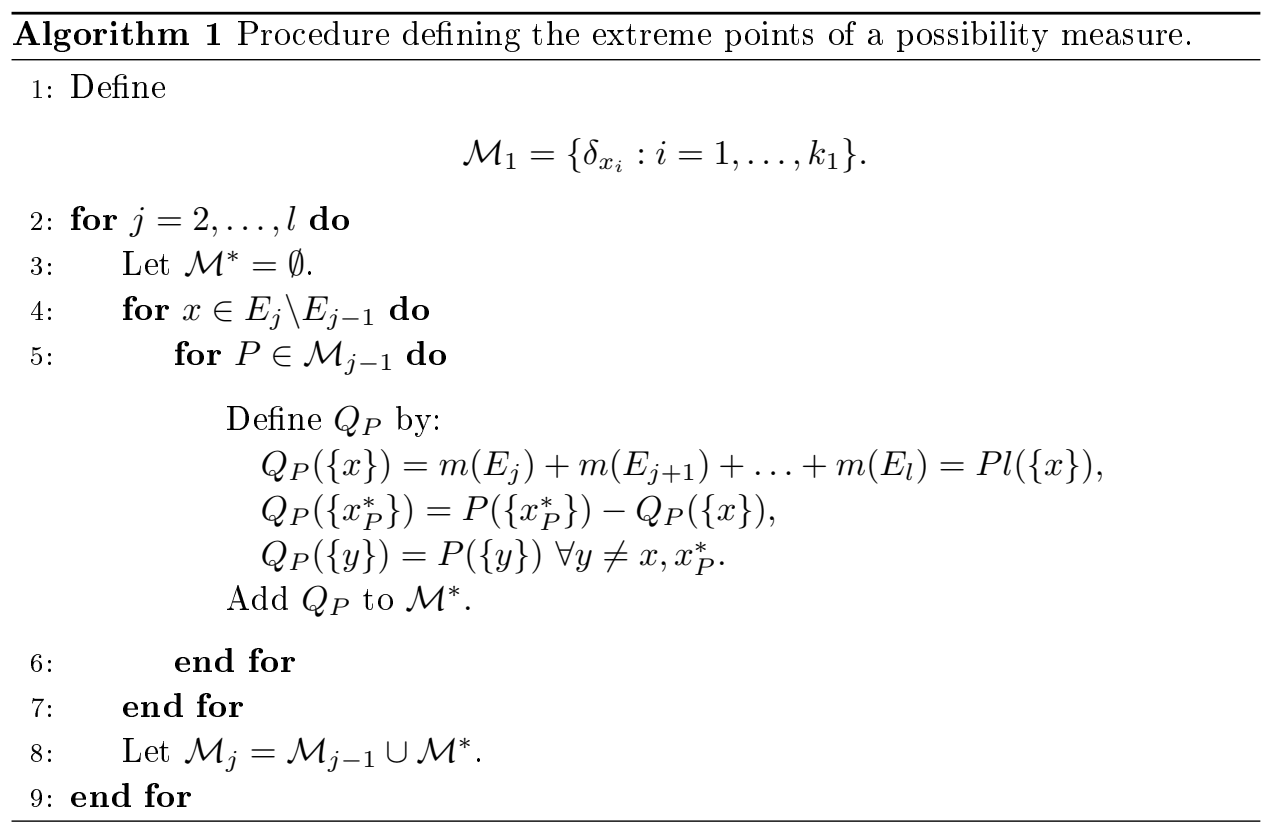

Example 1. Consider $\mathcal{X}=\left\{x_{1}, x_{2}, x_{3}, x_{4}, x_{5}\right\}$ and a possibility measure $\Pi$ with focal sets

$$
E_{1}=\left\{x_{1}, x_{2}\right\}, \quad E_{2}=\left\{x_{1}, x_{2}, x_{3}\right\}, \quad E_{3}=\mathcal{X}
$$

with masses $0.3,0.5$ and 0.2 , respectively. In the first step, we define the set $\mathcal{M}_{1}$ :

$$
\mathcal{M}_{1}=\{(1,0,0,0,0),(0,1,0,0,0)\} .
$$

For $j=2$, the only value in $E_{2} \backslash E_{1}$ is $x_{3}$. Then, we obtain the following probabilities: $(0.3,0,0.7,0,0)$ and $(0,0.3,0.7,0,0)$. Therefore:

$$
\mathcal{M}_{2}=\{(1,0,0,0,0),(0,1,0,0,0),(0.3,0,0.7,0,0),(0,0.3,0.7,0,0)\} .
$$

For $j=3$, we have to consider the elements $x_{4}$ and $x_{5}$. For $x_{4}$ we obtain the probabilities $(0.8,0,0,0.2,0),(0,0.8,0,0.2,0),(0.3,0,0.5,0.2,0)$ and $(0,0.3,0.5,0.2,0)$, and while for $x_{5}$ we obtain $(0.8,0,0,0,0.2),(0,0.8,0,0,0.2),(0.3,0,0.5,0,0.2)$ and $(0,0.3,0.5,0,0.2)$.

If we add these to the set $\mathcal{M}_{2}$ we obtain the 12 extreme points of $\mathcal{M}(\Pi)$.

Remark 1. Plausibility functions, and as a consequence also possibility measures, can be represented by means of measurable multi-valued mappings, or random sets ${ }^{4,19}$. In particular, a possibility measure on $\mathcal{P}(\mathcal{X})$ can always be obtained as the upper probability induced by a consonant random set, i.e., one with nested images ${ }^{13,20}$. It can be checked that the extreme points of the core of a possibility measure can be obtained as distributions induced by some measurable selections of the random set, and this idea is present in some of the results established in ${ }^{5,22}$. 
In terms of random sets, the focal elements of the possibility measure are the images that are attained with positive probability. Then the interpretation of the results in Corollary 1 is that the maximum number of extreme points is attained if and only if there is some order $\left\{x_{\sigma(1)}, x_{\sigma(2)}, \ldots, x_{\sigma(n)}\right\}$ in $\mathcal{X}$ such that the images that the associated random set takes with positive probability are $\left\{x_{\sigma(1)}, \ldots, x_{\sigma(i)}\right\}$ for $i=2, \ldots, n$, and possibly also $\left\{x_{\sigma(1)}\right\}$.

\section{Extreme points for maxitive $p$-boxes}

Next we consider another imprecise probability model that is also related to possibility measures and plausibility functions: $p$-boxes, both uni- and bivariate. In the univariate case, we shall consider a finite and ordered set $\mathcal{X}=\left\{x_{1}, \ldots, x_{n}\right\}$ with $x_{1}<\ldots<x_{n}$. When dealing with bivariate $p$-boxes, we shall consider two finite and ordered spaces $\mathcal{X}=\left\{x_{1}, \ldots, x_{n}\right\}$ and $\mathcal{Y}=\left\{y_{1}, \ldots, y_{m}\right\}$, where $x_{1}<\ldots<x_{n}$ and $y_{1}<\ldots<y_{m}$, and shall denote by $\mathcal{X} \times \mathcal{Y}$ their cartesian product. We shall assume without loss of generality that $n \leq m$.

\subsection{P-boxes}

Probability boxes ( $p$-boxes, for short) ${ }^{9}$ can be used to model the imprecise information about a (uni- or bi-variate) distribution function, by means of a lower and an upper functional. As such, they constitute an imprecise probability model. Their main advantage over other models is that they are computationally quite simple, being determined by point functions instead of set-valued ones.

Definition 2. A (univariate) $p$-box ${ }^{9}(\underline{F}, \bar{F})$ on $\mathcal{X}$ is a pair of increasing functions $\underline{F}, \bar{F}: \mathcal{X} \rightarrow[0,1]$ such that $\underline{F}\left(x_{n}\right)=\bar{F}\left(x_{n}\right)=1$. A bivariate $p$-box ${ }^{21}(\underline{F}, \bar{F})$ on $\mathcal{X} \times \mathcal{Y}$ is a pair of component-wise increasing functions $\underline{F}, \bar{F}: \mathcal{X} \times \mathcal{Y} \rightarrow[0,1]$ such that $\underline{F}\left(x_{n}, y_{m}\right)=\bar{F}\left(x_{n}, y_{m}\right)=1$.

A univariate $p$-box can be used to model the imprecise knowledge about the distribution function of a random variable $X$ taking values in the possibility space $\mathcal{X}$, and a bivariate $p$-box can be used to model the imprecise knowledge about the joint distribution function of two variables $X, Y$ taking values in $\mathcal{X}$ and $\mathcal{Y}$, respectively. We shall assume that these two variables are logically independent, so that the bivariate random variable $(X, Y)$ may take any value in the product space $\mathcal{X} \times \mathcal{Y}$.

Given a univariate $p$-box $(\underline{F}, \bar{F})$ on $\mathcal{X}$, its associated credal set is given by $\mathcal{M}(\underline{F}, \bar{F}):=\left\{P: \underline{F} \leq F_{P} \leq \bar{F}\right\}$, where $F_{P}$ denotes the cumulative distribution function associated with $P$. It can be used to determine the lower and upper probabilities associated with a $p$-box, given by ${ }^{10,25}$ :

$$
\begin{array}{ll}
\underline{P}(A)=\inf \left\{P(A) \mid \underline{F} \leq F_{P} \leq \bar{F}\right\} & \forall A \subseteq \mathcal{X}, \\
\bar{P}(A)=\sup \left\{P(A) \mid \underline{F} \leq F_{P} \leq \bar{F}\right\} & \forall A \subseteq \mathcal{X} .
\end{array}
$$


It was proven in ${ }^{25}$ that $\underline{P}, \bar{P}$ are a belief and a plausibility function, respectively. As a consequence, they are determined by the focal elements of their associated basic probability assignment. It can be checked that for every focal set $E$, it holds that $E=\{x \in \mathcal{X}: \min E \leq x \leq \max E\}$. Moreover, for any pair of focal sets $E_{1}, E_{2}$, it holds that either $\min E_{1} \leq \min E_{2}$ and $\max E_{1} \leq \max E_{2}$ or $\min E_{1} \geq \min E_{2}$ and $\max E_{1} \geq \max E_{2}$. We refer to ${ }^{6,10}$ for more information.

From ${ }^{16}$, the set $\mathcal{M}(\underline{F}, \bar{F})$ associated with a univariate $p$-box has at most $\mathcal{P}_{n}$ extreme points, where $\mathcal{P}_{n}$ is the Pell number, defined recursively by:

$$
\mathcal{P}_{0}=0, \quad \mathcal{P}_{1}=1, \quad \mathcal{P}_{n}=2 \mathcal{P}_{n-1}+\mathcal{P}_{n-2} .
$$

Those univariate $p$-boxes for which this maximal number of extreme points is attained were also investigated in ${ }^{16}$.

The upper probability associated with a univariate $p$-box by means of Equation (5) may not be a possibility measure. The connection between univariate $p$ boxes and possibility measures is clarified in the following proposition:

Proposition 3. ${ }^{26}$ Let $(\underline{F}, \bar{F})$ be a univariate $p$-box on $\mathcal{X}$. Its associated upper probability is a possibility measure if and only if $\underline{F}$ or $\bar{F}$ is vacuous, meaning that $\underline{F}=I_{\left\{x_{n}\right\}}$ or $\bar{F}=1$.

Thus, $p$-boxes inducing a possibility measure can be used to model situations where all the available information about an unknown cumulative distribution function is determined by either an upper bound (when $\underline{F}=I_{\left\{x_{n}\right\}}$ ) or a lower one (when $\bar{F}=1)$.

Next we focus on bivariate $p$-boxes, that were investigated in detail in ${ }^{18,21}$. For $i \in\{1, \ldots, n\}$ and $j \in\{1, \ldots, m\}$, the cumulative rectangle $A_{\left(x_{i}, y_{j}\right)}$ is given by

$$
A_{\left(x_{i}, y_{j}\right)}:=\left\{\left(x_{r}, y_{s}\right): r \leq i, s \leq j\right\} .
$$

The bivariate $p$-box $(\underline{F}, \bar{F})$ allows us to define the following upper probabilities:

$$
\bar{P}\left(A_{\left(x_{i}, y_{j}\right)}\right)=\bar{F}\left(x_{i}, y_{j}\right), \bar{P}\left(A_{\left(x_{i}, y_{j}\right)}^{c}\right)=1-\underline{F}\left(x_{i}, y_{j}\right),
$$

and using conjugacy, also the lower probabilities of the sets $A_{\left(x_{i}, y_{j}\right)}, A_{\left(x_{i}, y_{j}\right)}^{c}$. Then the credal set associated with the bivariate $p$-box is given by

$$
\begin{aligned}
& \mathcal{M}(\underline{F}, \bar{F})= \\
& \quad\left\{P: \underline{F}\left(x_{i}, y_{j}\right)=\underline{P}\left(A_{\left(x_{i}, y_{j}\right)}\right) \leq P\left(A_{\left(x_{i}, y_{j}\right)}\right) \leq \bar{P}\left(A_{\left(x_{i}, y_{j}\right)}\right)=\bar{F}\left(x_{i}, y_{j}\right) \forall i, j\right\} .
\end{aligned}
$$

However, $\underline{P}, \bar{P}$ need not coincide with the lower and the upper envelope of the set $\mathcal{M}(\underline{F}, \bar{F})$, meaning that they are not coherent ${ }^{27}$ lower and upper probabilities, respectively. This problem was investigated in ${ }^{21}$. It follows that $\underline{P}, \bar{P}$ are not belief and plausibility functions in general.

In ${ }^{17}$, we studied under which conditions the functions $\underline{P}, \bar{P}$ given by Equation (7), and extended to $\mathcal{P}(X \times Y)$ by taking the envelopes of $\mathcal{M}(\underline{F}, \bar{F})$, are a 
necessity and a possibility measure, respectively. We assumed the following technical condition:

$$
\bar{P}\left(\left\{\left(x_{i}, y_{j}\right)\right\}\right)>0 \quad \forall i=1, \ldots, n ; j=1, \ldots, m .
$$

This condition means that any element in $\mathcal{X} \times \mathcal{Y}$ belongs to at least one focal set, and it also helps simplifying some of the proofs. Therefore, from now on, whenever we consider the upper probability associated with a bivariate $p$-box defined on Equation $(7)$ and extended to $\mathcal{P}(\mathcal{X} \times \mathcal{Y})$ by computing the lower envelope of $\mathcal{M}(\underline{F}, \bar{F})$, we shall assume it to satisfy restriction (8).

We also established the following definitions:

Definition 3. ${ }^{17}$ A bivariate $p$-box $(\underline{F}, \bar{F})$ is said to be of type-1 when $\underline{F}$ is vacuous $\left(\underline{F}(x, y)=I_{\left\{\left(x_{n}, y_{m}\right)\right\}}(x, y) \forall(x, y) \in \mathcal{X} \times \mathcal{Y}\right)$, and is said to be of type-2 when it is not of type 1 and $\bar{F}$ is constant on $1(\bar{F}(x, y)=1 \forall(x, y) \in \mathcal{X} \times \mathcal{Y})$.

Similarly to the univariate case, type-1 (respectively, type-2) bivariate $p$-boxes can be used to model situations where the only available information about an unknown bivariate cumulative distribution function is given by an upper (respectively, a lower) bound.

We call a bivariate $p$-box maxitive when the upper probability $\bar{P}$ it induces by means of Equation (7) is a possibility measure. We have the following characterization:

Theorem 2. ${ }^{17}$ Let $(\underline{F}, \bar{F})$ be a bivariate p-box satisfying Equation (8). Its associated upper probability is maxitive if and only if one of the following conditions hold:

Condition $1(\underline{F}, \bar{F})$ is of type-1, $\bar{F}\left(x_{i}, y_{j}\right)=\max \left\{\bar{F}\left(x_{i}, y_{1}\right), \bar{F}\left(x_{1}, y_{j}\right)\right\}$ for any $i=1, \ldots, n ; j=1, \ldots, m$ and either

(a) $\bar{F}\left(x, y_{m}\right)=1$ for any $x \in \mathcal{X}$; or

(b) $\bar{F}\left(x_{n}, y\right)=1$ for any $y \in \mathcal{Y}$.

Condition $2(\underline{F}, \bar{F})$ is of type-2 and $\underline{F}\left(x_{i}, y_{j}\right)=\min \left\{\underline{F}\left(x_{i}, y_{m}\right), \underline{F}\left(x_{n}, y_{j}\right)\right\}$ for any $i=1, \ldots, n ; j=1, \ldots, m$.

Remark 2. Interestingly, maxitive $p$-boxes can be embedded into the more general framework of generalized $p$-boxes ${ }^{6}$, that extend $p$-boxes to not necessarily ordered spaces $\mathcal{Z}$. Given such a space $\mathcal{Z}$ : a generalized $p$-box is a pair of functions $\underline{F}, \bar{F}$ : $\mathcal{Z} \rightarrow[0,1]$ that are comonotone (they increase and decrease simultaneously) and componentwise ordered: $\underline{F} \leq \bar{F}$.

In the case of maxitive bivariate $p$-boxes, Theorem 2 tells us that either $\underline{F}$ is vacuous or $\bar{F}$ is constant on 1 ; in any of the two cases, it trivially follows that $\underline{F}$ and $\bar{F}$ are comonotone and componentwise ordered, and as a consequence maxitive bivariate $p$-boxes are in particular generalized $p$-boxes; this could be of interest because, even if we assume that $\mathcal{X}, \mathcal{Y}$ are totally ordered spaces, they only induce a partial order on the product space $\mathcal{X} \times \mathcal{Y}$. 
Although one may think that the extreme points for maxitive bivariate $p$-boxs could be computed using the results established in ${ }^{16}$ for generalized $p$-boxes, this will not be the case in general. The reason is that the bivariate structure of maxitive bivariate $p$-boxes is not preserved when we study them as generalized $p$-boxes, since there is not a one-to-one correspondence between their credal sets. To see this more clearly, consider a type-1 maxitive bivariate $p$-box defined by:

$$
\begin{array}{c|cccc} 
& \left(x_{1}, y_{1}\right) & \left(x_{1}, y_{2}\right) & \left(x_{2}, y_{1}\right) & \left(x_{2}, y_{2}\right) \\
\hline \bar{F} & 0 & 0 & 0 & 1 \\
0.6 & 1 & 1 & 1
\end{array}
$$

If we define the four-element space $Z$ by:

$$
z_{1}=\left(x_{1}, y_{1}\right), \quad z_{2}=\left(x_{1}, y_{2}\right), \quad z_{3}=\left(x_{2}, y_{1}\right), \quad z_{4}=\left(x_{2}, y_{2}\right),
$$

we obtain the generalized $p$-box $\left(\underline{F}_{Z}, \bar{F}_{Z}\right)$ given by:

$$
\begin{array}{c|cccc} 
& z_{1} & z_{2} & z_{3} & z_{4} \\
\hline \bar{F}_{Z} & 0 & 0 & 0 & 1 \\
\bar{F}_{Z} & 0.6 & 1 & 1 & 1
\end{array}
$$

Although $\bar{F}_{Z}$ is a univariate cumulative distribution function, when we consider our bivariate framework we obtain the function $\bar{F}$ that is not a bivariate cumulative distribution function, because it does not satisfy the rectangle inequality. This shows that the representation as a generalized $p$-boxes is entailing a loss of information in this context.

In the remainder of the section we investigate the number of extreme points of the cores of maxitive $p$-boxes. We consider both the univariate and bivariate cases and, in this second case, type- 1 and type- 2 bivariate $p$-boxes.

\subsection{Maxitive univariate $p$-boxes}

By Proposition 3 , a univariate $p$-box $(\underline{F}, \bar{F})$ defines a maxitive function if and only if either $\underline{F}=I_{\left\{x_{n}\right\}}$ or $\bar{F}=1$. In order to compute the extreme points of the core of $(\underline{F}, \bar{F})$, it will be helpful to look at the form of the focal sets.

(a) Assume that $\underline{F}=I_{\left\{x_{n}\right\}}$. In that case, denote by $\left\{u_{1}, \ldots, u_{k}\right\}=\left\{x_{i} \in \mathcal{X} \mid\right.$ $\left.\bar{F}\left(x_{i}\right)>\bar{F}\left(x_{i-1}\right)\right\}$, where $\bar{F}\left(x_{0}\right):=0$ and $u_{1}<\ldots<u_{k}$. Then, the focal sets are given by:

$$
E_{1}=\left\{x \in \mathcal{X} \mid x \geq u_{1}\right\}, \ldots, E_{k}=\left\{x \in \mathcal{X} \mid x \geq u_{k}\right\},
$$

with respective masses $m\left(E_{1}\right)=\bar{F}\left(u_{1}\right), m\left(E_{i}\right)=\bar{F}\left(u_{i}\right)-\bar{F}\left(u_{i-1}\right)$ for $i=2, \ldots, k$. It holds that $E_{1} \supset E_{2} \supset \ldots \supset E_{k}$.

(b) Assume that $\bar{F}=1$. Denote by $\left\{u_{1}, \ldots, u_{k}\right\}=\left\{x_{i} \in \mathcal{X} \mid \underline{F}\left(x_{i}\right)>\underline{F}\left(x_{i-1}\right)\right\}$, where $\underline{F}\left(x_{0}\right)=0$ and $u_{1}<\ldots<u_{k}$. The focal sets are given by:

$$
E_{1}=\left\{x \in \mathcal{X} \mid x \leq u_{1}\right\}, \ldots, E_{k}=\left\{x \in \mathcal{X} \mid x \leq u_{k}\right\},
$$


with masses $m\left(E_{1}\right)=\underline{F}\left(u_{1}\right), m\left(E_{i}\right)=\underline{F}\left(u_{i}\right)-\underline{F}\left(u_{i-1}\right)$ for any $i=2, \ldots, k$. It holds that $E_{1} \subset \ldots \subset E_{k}$.

Using the above expressions of the focal sets, we easily see that the plausibility function associated with a maxitive univariate $p$-box may have at most $n$ different focal sets:

(a) If $\underline{F}=I_{\left\{x_{n}\right\}}$, take $\bar{F}$ such that $0<\bar{F}\left(x_{1}\right)<\ldots<\bar{F}\left(x_{n}\right)=1$. Then the focal sets are $E_{1}=\mathcal{X}, E_{2}=\left\{x_{2}, x_{3}, \ldots, x_{n}\right\}, \ldots, E_{n}=\left\{x_{n}\right\}$.

(b) If $\bar{F}=1$, take $\underline{F}$ such that $0<\underline{F}\left(x_{1}\right)<\ldots<\underline{F}\left(x_{n}\right)=1$. Then the focal sets are given by $E_{1}=\left\{x_{1}\right\}, E_{2}=\left\{x_{1}, x_{2}\right\}, \ldots, E_{n}=\mathcal{X}$.

In both cases, using Equation (3), we obtain that $k_{i}=1$ for any $i=1, \ldots, n$, and therefore the number of extreme points equals $1 \cdot(1+1)^{n-1}=2^{n-1}$.

This shows that given a maxitive univariate $p$-box $(\underline{F}, \bar{F})$, its associated credal set has at most $2^{n-1}$ extreme points. This number is smaller than the maximum number of extreme points of the credal set associated with an arbitrary univariate $p$-box, which is the Pell number ${ }^{16}$, and it is equal to the maximal number of extreme points of the credal set associated with a possibility measure.

\subsection{Type-2 maxitive bivariate p-boxes}

We turn now to the bivariate case. By Theorem 2, any maxitive bivariate $p$-box must be either of type-1 (meaning that the lower distribution function $F$ is equal to $I_{\left(x_{n}, y_{m}\right)}$ ) or of type-2 (meaning that the upper distribution function $\bar{F}$ is constant on 1). We begin by considering this second case.

Consider thus a type-2 maxitive bivariate $p$-box $(\underline{F}, \bar{F})$ on $\mathcal{X} \times \mathcal{Y}$, and let $\Pi$ denote its associated possibility measure. In order to alleviate the notation, we shall refer to $\Pi$ as a type-2 possibility measure on $\mathcal{P}(\mathcal{X} \times \mathcal{Y})$. From ${ }^{17}$, the focal sets of $\Pi$ are given by:

$$
E_{1}=A_{\left(v_{1}, w_{1}\right)}, \ldots, E_{l}=A_{\left(v_{l}, w_{l}\right)}=\mathcal{X} \times \mathcal{Y},
$$

for some elements of $\mathcal{X}$ and $\mathcal{Y} v_{1} \leq \ldots \leq v_{l}=x_{n}$ and $w_{1} \leq \ldots \leq w_{l}=y_{m}$, respectively, and where $A_{\left(v_{j}, w_{j}\right)}$ is the cumulative rectangle determined by the pair $\left(v_{j}, w_{j}\right)$ defined on Equation (6).

Our first result provides an upper bound of the maximal number of extreme points of $\mathcal{M}(\Pi)$.

Proposition 4. Let $\Pi$ be a type-2 possibility measure on $\mathcal{P}(\mathcal{X} \times \mathcal{Y})$. If $n, m>1$, then the number of extreme points of $\mathcal{M}(\Pi)$ is strictly smaller than $2^{\text {nm-1 }}$.

Thus, unlike the univariate case, the cores of the possibility measures associated with maxitive type-2 $p$-boxes will have in general fewer extreme points that those associated with arbitrary possibility measures on $\mathcal{P}(\mathcal{X} \times \mathcal{Y})$. The key here is that the possibility measure induced by a maxitive type- $2 p$-box cannot take different values on all elements of $\mathcal{X} \times \mathcal{Y}$. 
In this section, we shall establish the tightest upper bound on the number of extreme points of $\mathcal{M}(\Pi)$. By Propositions 1 and 2, this maximal upper bound can only be attained when it is not possible to add an intermediate cumulative rectangle between two consecutive focal sets. In other words, if $E_{1} \subset \ldots \subset E_{l}$ are the focal sets of $\Pi$, there cannot exist a cumulative rectangle $A_{(x, y)}$ such that $E_{i} \subset A_{(x, y)} \subset E_{i+1}$ for some $i=1, \ldots, l-1$.

It also follows that the smallest focal element $E_{1}=A_{\left(v_{1}, w_{1}\right)}$ must be one of $A_{\left(x_{1}, y_{1}\right)}, A_{\left(x_{1}, y_{2}\right)}$ or $A_{\left(x_{2}, y_{1}\right)}$, while for any other focal element $E_{i}=A_{\left(v_{i}, w_{i}\right)}$ with $i>2$, one of the following conditions must hold:

(C1) $v_{i}=v_{i-1}$ and $w_{i}, w_{i-1}$ are consecutive elements of $\mathcal{X}$.

(C2) $w_{i}=w_{i-1}$ and $v_{i}, v_{i-1}$ are consecutive elements of $\mathcal{Y}$.

This implies that for $\mathcal{M}(\Pi)$ to have the maximal number of extreme points, it is necessary that one of the following conditions holds:

(Type 2-a) $\Pi$ has $n+m-1$ focal sets $E_{1} \subset \ldots \subset E_{n+m-1}$, where $E_{1}=A_{\left(x_{1}, y_{1}\right)}$ and for any $i=2, \ldots, n+m-1, E_{i}$ and $E_{i-1}$ satisfy either (C1) or (C2);

(Type 2-b) $\Pi$ has $n+m-2$ focal sets $E_{2} \subset \ldots \subset E_{n+m-1}$, where $E_{2}$ is either $A_{\left(x_{2}, y_{1}\right)}$ or $A_{\left(x_{1}, y_{2}\right)}$. Also, for any $i=3, \ldots, n+m-1, E_{i}$ and $E_{i-1}$ satisfy either (C1) or (C2).

This motivates the following definition:

Definition 4. A type-2 possibility measure $\Pi$ will be called optimal when it satisfies either (Type2-a) or (Type2-b). In order to unify the notation, we shall always denote the focal sets of $\Pi$ by $E_{1} \subset E_{2} \subset \ldots \subset E_{n+m-1}$, and make $E_{1}=\emptyset$ and $m\left(E_{1}\right)=0$ when we are in case (Type2-b).

Taking into account the previous notation and comments, if $\Pi$ is an optimal type-2 possibility measure with focal sets $E_{1} \subset \ldots \subset E_{n+m-1}$, then

$$
E_{j}=A_{\left(x_{s}, y_{r}\right)} \Rightarrow j=r+s-1 \quad \forall j=2, \ldots, n+m-1 .
$$

Therefore, $r+s$ is even (respectively, odd) whenever $j$ is odd (respectively, even).

Interestingly, not all optimal type- 2 possibility measures have the same number of extreme points:

Example 2. Consider two optimal type-2 possibilities $\Pi_{1}, \Pi_{2}$ on $\mathcal{P}\left(\left\{x_{1}, x_{2}, x_{3}\right\} \times\right.$ $\left.\left\{y_{1}, y_{2}\right\}\right)$ with respective focal sets:

$$
\begin{array}{ll}
E_{1}=A_{\left(x_{1}, y_{1}\right)}, & E_{2}=A_{\left(x_{1}, y_{2}\right)}, E_{3}=A_{\left(x_{2}, y_{2}\right)}, E_{4}=A_{\left(x_{3}, y_{2}\right)}, \text { for } \Pi_{1}, \\
E_{1}^{\prime}=A_{\left(x_{1}, y_{1}\right)}, & E_{2}^{\prime}=A_{\left(x_{2}, y_{1}\right)}, E_{3}^{\prime}=A_{\left(x_{3}, y_{1}\right)}, E_{4}^{\prime}=A_{\left(x_{3}, y_{2}\right)}, \text { for } \Pi_{2} .
\end{array}
$$

Taking Equation (3) into account, we obtain $k_{1}=1, k_{2}=1, k_{3}=2, k_{4}=2$ for $\Pi_{1}$ and $k_{1}^{\prime}=1, k_{2}^{\prime}=1, k_{3}^{\prime}=1, k_{4}^{\prime}=3$ for $\Pi_{2}$. Then, the number of extreme points of $\mathcal{M}\left(\Pi_{1}\right)$ is:

$$
k_{1}\left(1+k_{2}\right)\left(1+k_{3}\right)\left(1+k_{4}\right)=1 \cdot 2 \cdot 3 \cdot 3=18,
$$


while the number of extreme points of $\mathcal{M}\left(\Pi_{2}\right)$ is:

$$
k_{1}^{\prime}\left(1+k_{2}^{\prime}\right)\left(1+k_{3}^{\prime}\right)\left(1+k_{4}^{\prime}\right)=1 \cdot 2 \cdot 2 \cdot 4=16 .
$$

We see that the maximal number of extreme points for a type-2 possibility measure is not determined by looking at an arbitrary optimal one; we must instead determine which configuration generates the maximal number of extreme points, and also this maximal number. For this aim, we introduce the following notion.

Definition 5. Let $\Pi$ be an optimal type-2 possibility measure on $\mathcal{P}(\mathcal{X} \times \mathcal{Y})$ with focal sets $E_{1} \subset \ldots \subset E_{n+m-1}$. The level of the focal set $E_{j}=A_{\left(x_{r}, y_{s}\right)}$ is defined as $\left\lfloor\frac{|r-s|}{2}\right\rfloor$, that is, the smallest integer less than or equal to $\frac{|r-s|}{2}$.

Equivalently, we say that $E_{j}=A_{\left(x_{r}, y_{s}\right)}$ has level $i$ when:

(a) $|r-s|=2 i$, if $r+s$ is even.

(b) $|r-s|=2 i+1$, if $r+s$ is odd.

Next we show some properties of the levels of the focal sets.

Proposition 5. Let $\Pi$ be an optimal type-2 possibility measure on $\mathcal{P}(\mathcal{X} \times \mathcal{Y})$ with focal sets $E_{1} \subset \ldots \subset E_{n+m-1}$.

(i) $\operatorname{level}\left(E_{2 j+1}\right) \geq \max \left\{\right.$ level $\left(E_{2 j+2}\right)$, level $\left.\left(E_{2 j}\right)\right\}$ for every $j$.

(ii) Let $E_{2 j}=A_{\left(x_{r}, y_{s}\right)}$ for $j \geq 1$, where $r<s$. If level $\left(E_{2 j}\right)=i$, then:

$$
k_{2 j+1}= \begin{cases}s & \text { if level }\left(E_{2 j+1}\right)=i . \\ r & \text { if level }\left(E_{2 j+1}\right)=i+1 .\end{cases}
$$

(iii) Let $E_{2 j+1}=A_{\left(x_{r}, y_{s}\right)}$ for $j \geq 1$, where $r \leq s$. If level $\left(E_{2 j+1}\right)=i$, then:

$$
k_{2 j+2}= \begin{cases}r & \text { if level }\left(E_{2 j+1}\right)=i . \\ s & \text { if level }\left(E_{2 j+1}\right)=i-1 .\end{cases}
$$

We deduce that for any optimal type-2 possibility measure $\Pi$, the number of extreme points of $\mathcal{M}(\Pi)$ is determined by the levels of the focal sets; note that, even if the second and third statements assume that $r \leq s$, in a similar manner we can establish their analog when $r \geq s$ (in the case of the second statement note that for $E_{2 j}=A_{\left(x_{r}, y_{s}\right)}$ we cannot have $r=s$, because of Equation (9)).

On the other hand, Proposition 5, together with conditions (C1)-(C2), imply that the levels satisfy the following properties:

(L1) level $\left(E_{2 j+1}\right)-\operatorname{level}\left(E_{2 j}\right) \in\{0,1\} \forall j$.

(L2) $\left|\operatorname{level}\left(E_{j}\right)-\operatorname{level}\left(E_{j+2}\right)\right| \leq 1$ for any $j=1, \ldots, n+m-3$.

This allows to give a graphical representation of the optimal type- 2 possibility measures. In Figure 1 we have depicted the case $n=3, m=5$. There, the element $(i, j)$ refers to the focal set $A_{(i, j)}$. The optimal type-2 possibilities start at $(1,1)$ 
and follow the arrows until $(3,5)$. There are as many optimal type-2 possibilities as different paths to $(3,5)$. Note that in this picture we have considered the case $E_{1}=A_{\left(x_{1}, y_{1}\right)}$; the case of (Type2-b) is determined with a starting point in either $(2,1)$ or $(1,2)$.

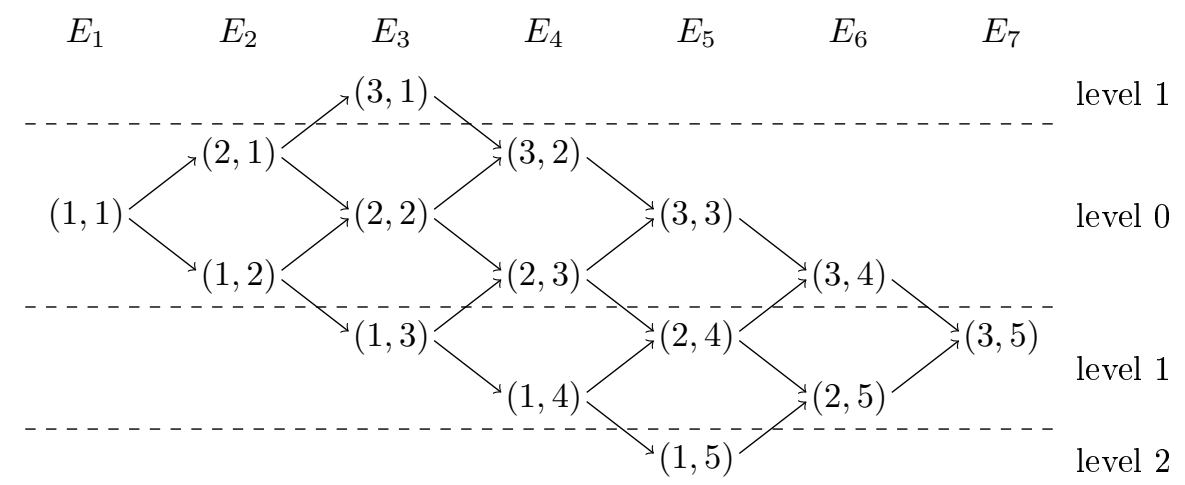

Fig. 1: Optimal type-2 possibility measures for $n=3, m=5$. The element $(i, j)$ refers to the focal set $A_{\left(x_{i}, y_{j}\right)}$.

On the other hand, if we fix the level of the focal set $E_{i}$, there are only two possible focal sets with this level, and we can work without loss of generality with one of them. This is clarified in the following proposition:

Proposition 6. Let $\Pi, \Pi^{\prime}$ be two optimal type-2 possibility measures on $\mathcal{P}(\mathcal{X} \times \mathcal{Y})$ with respective focal sets $E_{1} \subset \ldots \subset E_{n+m-1}$ and $E_{1}^{\prime} \subset \ldots \subset E_{n+m-1}^{\prime}$. Assume $E_{i}=A_{\left(x_{r_{i}}, y_{s_{i}}\right)}$ and $E_{i}^{\prime}=A_{\left(x_{r_{i}^{\prime}}, y_{s_{i}^{\prime}}\right)}$ for $i=1, \ldots, m+1$.

(i) If level $\left(E_{i}\right)=\operatorname{level}\left(E_{i}^{\prime}\right)$ then $\min \left\{r_{i}, s_{i}\right\}=\min \left\{r_{i}^{\prime}, s_{i}^{\prime}\right\}$ and $\max \left\{r_{i}, s_{i}\right\}=$ $\max \left\{r_{i}^{\prime}, s_{i}^{\prime}\right\}$.

(ii) Define $E_{i}^{\prime \prime}:=A_{\left(x_{\min \left\{r_{i}, s_{i}\right\}}, y_{\max \left\{r_{i}, s_{i}\right\}}\right)}$ for $i=1, \ldots, n+m-1$. Then the sets $E_{i}^{\prime \prime}$ are nested and their associated possibility measure $\Pi^{\prime \prime}$ is such that $\mathcal{M}(\Pi)$ and $\mathcal{M}\left(\Pi^{\prime \prime}\right)$ have the same number of extreme points.

Taking this result into account, we shall assume without loss of generality that for any optimal type- 2 possibility measure $\Pi$ with focal sets $E_{1} \subset \ldots \subset E_{n+m-1}$, it holds that $E_{i}=A_{\left(x_{r}, y_{s}\right)}$ with $r \leq s$ for every $i=1, \ldots, n+m-1$. This will simplify some of the proofs of the upcoming results.

We are finally ready to establish the main results of this subsection.

Proposition 7. Let $\Pi$ and $\Pi^{\prime}$ be two optimal type-2 possibility measures on $\mathcal{P}(\mathcal{X} \times$ $\mathcal{Y})$ with respective focal sets $E_{1} \subset \ldots \subset E_{n+m-1}$ and $E_{1}^{\prime} \subset \ldots \subset E_{n+m-1}^{\prime}$. If level $\left(E_{j}\right) \leq \operatorname{level}\left(E_{j}^{\prime}\right) \forall j$, with strict inequality in at least one $j$, then $\mathcal{M}(\Pi)$ has more extreme points than $\mathcal{M}\left(\Pi^{\prime}\right)$. 
This result allows us to establish the maximal number of extreme points of a type-2 possibility measure, and to determine which configurations produce this maximal number.

Theorem 3. Let $\Pi$ be an optimal type-2 possibility measure on $\mathcal{P}(\mathcal{X} \times \mathcal{Y})$ with $n+m-1$ focal sets $E_{1} \subset \ldots \subset E_{n+m-1}$. Assume without loss of generality that $n \leq m$ (otherwise, we just need to switch the role of $n$ and $m$ ). Then, the maximal number of extreme points of $\mathcal{M}(\Pi)$ is

$$
\frac{(n !)^{2}(n+1)^{m-n+1}}{2},
$$

and this number is attained if and only if level $\left(E_{j}\right)=0$ for all $j=1, \ldots, 2 n$. On the other hand, the minimal number of extreme points for an optimal type-2 possibility is $2^{m-1}(m+1)^{n-1}$.

The proof of this theorem, that we can find in the Appendix, details which optimal type-2 possibilities attain the maximum number of extreme points. They can be obtained by moving alternatively through the two axis, giving rise thus to the following focal sets:

$$
\begin{aligned}
& E_{1}=\left(x_{1}, y_{1}\right), \\
& E_{2 j}=\left(x_{j+1}, y_{j}\right) \text { or } E_{2 j}=\left(x_{j}, y_{j+1}\right), \quad \forall j=1, \ldots, n-1, \\
& E_{2 j+1}=\left(x_{j+1}, y_{j+1}\right), \quad \forall j=1, \ldots, n-1,
\end{aligned}
$$

and if $m>n$,

$$
E_{2 n-1+j}=\left(x_{n}, y_{n+j}\right), \quad \forall j=1, \ldots, m-n .
$$

In a similar manner, from the same proof we obtain a procedure to build optimal type-2 possibilities that attain the minimum number of extreme points. Here there are two possible scenarios (remember that we are assuming throughout that $n \leq m$ ):

- If $n<m$, the focal sets are:

$$
E_{j}=A_{\left(x_{1}, y_{j}\right)} \forall j=1, \ldots, m, \quad E_{j}=A_{\left(x_{j-m+1}, y_{m}\right)} \forall j=m+1, \ldots, m+n-1 .
$$

- If $n=m$, the focal sets are either:

$$
\begin{aligned}
& E_{j}=A_{\left(x_{1}, y_{j}\right)} \forall j=1, \ldots, m, \quad E_{j}=A_{\left(x_{j-m+1}, y_{m}\right)} \forall j=m+1, \ldots, m+n-1 . \\
& \text { or: } \\
& E_{j}=A_{\left(x_{j}, y_{1}\right)} \forall j=1, \ldots, n, \quad E_{j}=A_{\left(x_{n}, y_{j-n+1}\right)} \forall j=n+1, \ldots, m+n-1 .
\end{aligned}
$$

Note that for non-optimal type-2 possibility measures the minimum number of extreme points if $n m$, and this value is attained when the only focal set of the associated plausibility function is $\mathcal{X} \times \mathcal{Y}$ (which as a consequence has mass 1 ). 
In the particular case when $n=1$, the smallest and greatest number of extreme points of the core of $(\underline{F}, \bar{F})$ are $m$ and $2^{m-1}$, respectively. These two correspond to the bounds for univariate $p$-boxes considered in Section 4.2.

In Figure 2 we consider again the case $n=3, m=5$. All the optimal type-2 possibilities that start in $(1,1),(2,1)$ or $(1,2)$ and move to $(3,5)$ only through red nodes and arrows are those with the maximal number of extreme points, in this case $\frac{(3 !)^{2} \cdot 4^{3}}{2}=1152$, by Equation $(10)$.

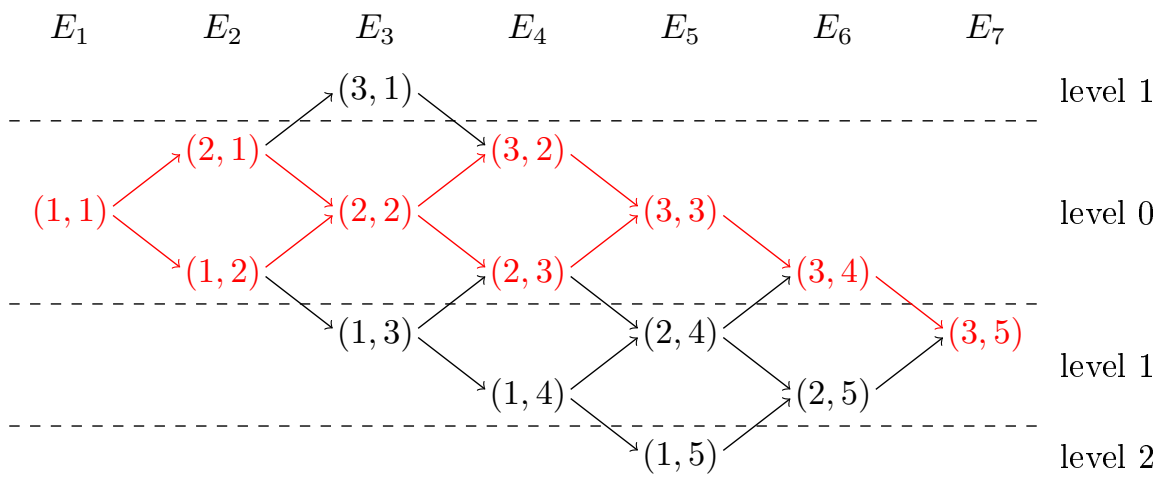

Fig. 2: Optimal type-2 possibility measures for $n=3, m=5$. Those attaining the maximal number of extreme points are the ones going from either $(1,1),(1,2)$ or $(2,1)$ to $(3,5)$ through red nodes and arrows.

Note that optimal type- 2 possibility measures are not in a different layer with respect to the number of extreme points they induce, in the sense that if $\Pi, \Pi^{\prime}$ are type- 2 possibility measures such that $\Pi$ is optimal and $\Pi^{\prime}$ is not, it does not necessarily hold that $|\operatorname{ext}(\mathcal{M}(\Pi))| \geq\left|\operatorname{ext}\left(\mathcal{M}\left(\Pi^{\prime}\right)\right)\right|$. This is because we can find non-optimal type-2 possibility measures with more than $2^{m-1}(m+1)^{n-1}$ extreme points:

Example 3. Consider $n=2$ and $m=5$, and the possibility measure $\Pi$ with focal sets:

$$
E_{1}=A_{\left(x_{2}, y_{2}\right)}, \quad E_{2}=A_{\left(x_{2}, y_{3}\right)}, \quad E_{3}=A_{\left(x_{2}, y_{4}\right)}, \quad E_{4}=A_{\left(x_{2}, y_{5}\right)} .
$$

Then $k_{1}=\left|E_{1}\right|=4, k_{2}=\left|E_{2} \backslash E_{1}\right|=2, k_{3}=\left|E_{3} \backslash E_{2}\right|=2$ and $k_{4}=\left|E_{4} \backslash E_{3}\right|=2$. By Theorem 1, the number of extreme points of $\mathcal{M}(\Pi)$ is:

$$
k_{1}\left(1+k_{2}\right)\left(1+k_{3}\right)\left(1+k_{4}\right)=4 \cdot 3 \cdot 3 \cdot 3=108 .
$$

This is strictly greater than the minimal number of extreme points of a type-2 possibility with $n+m-1$ focal elements, which according to Theorem 3 is $2^{m-1}(m+$ 1) ${ }^{n-1}=2^{4} \cdot 6=96$. 


\subsection{Type-1 maxitive bivariate p-boxes}

We consider in this subsection the possibility measures $\Pi$ generated by type- 1 maxitive bivariate $p$-boxes.

Definition 6. A possibility measure associated with a type-1 maxitive bivariate p-box will be called type-1 possibility measure.

From ${ }^{17}$, the focal sets of a type-1 possibility measure are given by:

$$
E_{1}=A_{\left(v_{l-1}, w_{l-1}\right)}^{c}, E_{2}=A_{\left(v_{l-2}, w_{l-2}\right)}^{c}, \ldots, E_{l-1}=A_{\left(v_{1}, w_{1}\right)}^{c}, E_{l}=\mathcal{X} \times \mathcal{Y},
$$

where $v_{1} \leq \ldots \leq v_{l-1}$ (resp., $w_{1} \leq \ldots \leq w_{l-1}$ ) are elements from $\mathcal{X}$ (resp., $\mathcal{Y}$ ). From Propositions 1 and 2, for $\Pi$ to determine a credal set with a maximal number of extreme points, there can be no set $E=A_{\left(x_{s}, y_{r}\right)}^{c}$ satisfying $E_{i} \subset E \subset E_{i+1}$ for some $i=1, \ldots, l-1$. This means that if $E_{i}=A_{\left(v_{i}, w_{i}\right)}$ is a focal set with $i>2$, one of the following conditions must hold:

(C'1) $v_{i}=v_{i-1}$ and $w_{i}, w_{i-1}$ are consecutive elements of $\mathcal{X}$.

$\left(\mathrm{C}^{\prime} 2\right) w_{i}=w_{i-1}$ and $v_{i}, v_{i-1}$ are consecutive elements of $\mathcal{Y}$.

Also, $\Pi$ must have $n+m-1$ focal sets and $\left(v_{1}, w_{1}\right)=\left(x_{1}, y_{1}\right)$. Therefore, a necessary condition for the core of $\Pi$ to attain the maximum number of extreme points is that its focal sets can be expressed by $E_{1} \subset \ldots \subset E_{n+m-1}=\mathcal{X} \times \mathcal{Y}, E_{n+m-2}=A_{\left(x_{1}, y_{1}\right)}^{c}$ and for any $i=2, \ldots, n+m-1$ either ( $\left.\mathrm{C}^{\prime} 1\right)$ or ( $\left.\mathrm{C}^{\prime} 2\right)$ holds. A type-1 possibility measure satisfying these properties will be called an optimal type-1 possibility.

According to Equation (4), the number of extreme points of $\mathcal{M}(\Pi)$ is:

$$
k_{1}\left(1+k_{2}\right) \ldots\left(1+k_{n+m-1}\right),
$$

where:

$$
k_{1}=\left|A_{\left(v_{l-1}, w_{l-1}\right)}^{c}\right|, \quad k_{i}=\left|E_{i} \backslash E_{i-1}\right| \quad \forall i=2, \ldots, n+m-1 .
$$

It is useful to note that there is a one-to-one correspondence between optimal type1 and (Type2-a) possibility measures. The reason is that for an optimal type-1 possibility measure with focal sets

$E_{1}=A_{\left(v_{1}, w_{1}\right)}^{c} \subset \ldots \subset E_{n+m-2}=A_{\left(v_{n+m-2}, w_{n+m-2}\right)}^{c}=A_{\left(x_{1}, y_{1}\right)}^{c} \subset E_{n+m-1}=\mathcal{X} \times \mathcal{Y}$, we can define an optimal type-2 possibility $\widetilde{\Pi}$ with focal sets:

$$
\widetilde{E}_{j}= \begin{cases}E_{n+m-2}^{c}=A_{\left(v_{n+m-2}, w_{n+m-2}\right)} & \text { for } j=1 \\ E_{n+m-j-1}^{c}=A_{\left(v_{n+m-j-1}, w_{n+m-j-1}\right)} & \text { for } j=2, \ldots, n+m-2 \\ \mathcal{X} \times \mathcal{Y} & \text { for } j=n+m-1 .\end{cases}
$$

Furthermore, if we denote by $k_{1}=\left|E_{1}\right|, k_{i}=\left|E_{i} \backslash E_{i-1}\right|$ for any $i=2, \ldots, n$, then:

$$
\begin{aligned}
& \widetilde{k}_{1}=\left|\widetilde{E}_{1}\right|=\left|A_{\left(v_{n+m-2}, w_{n+m-2}\right)}\right|=\left|A_{\left(x_{1}, y_{1}\right)}\right|=\left|\mathcal{X} \times \mathcal{Y} \backslash A_{\left(x_{1}, y_{1}\right)}^{c}\right|=k_{n+m-1} . \\
& \widetilde{k}_{i}=\left|\widetilde{E}_{i} \backslash \widetilde{E}_{i-1}\right|=\left|A_{\left(v_{n+m-i}, w_{n+m-i}\right)} \backslash A_{\left(v_{n+m-i+1}, w_{n+m-i+1}\right)}\right| \\
& =\left|A_{\left(v_{n+m-i+1}, w_{n+m-i+1}\right)}^{c} \backslash A_{\left(v_{n+m-i}, w_{n+m-i}\right)}^{c}\right|=\left|E_{n+m-i} \backslash E_{n+m-i-1}\right|=k_{n+m-i} .
\end{aligned}
$$


This means that the number of extreme points of an optimal type- 1 possibility is equal to $k_{1} \prod_{i=2}^{n+m-1}\left(1+k_{i}\right)$, or equivalently:

$$
\widetilde{k}_{n+m-1} \prod_{i=1}^{n+m-2}\left(1+\widetilde{k}_{i}\right)
$$

It follows from this that $\mathcal{M}(\Pi)$ and $\mathcal{M}(\widetilde{\Pi})$ will not have in general the same number of extreme points.

Example 4. The one-to-one correspondence between optimal type-1 and (Type2a) possibility measures defined in Equation (11) can be given a graphical interpretation. Consider, for simplicity, the case $n=2, m=3$, and the optimal type- 1 possibility measure whose focal sets are:

$$
E_{1}=A_{\left(x_{2}, y_{2}\right)}^{c}, \quad E_{2}=A_{\left(x_{1}, y_{2}\right)}^{c}, \quad E_{3}=A_{\left(x_{1}, y_{1}\right)}, \quad E_{4}=\mathcal{X} \times \mathcal{Y} .
$$

Then, according to the previous comments, its associated (Type2-a) possibility measure has the following focal sets:

$$
\widetilde{E}_{1}=A_{\left(x_{1}, y_{1}\right)}, \quad \widetilde{E}_{2}=A_{\left(x_{1}, y_{2}\right)}, \quad \widetilde{E}_{3}=A_{\left(x_{2}, y_{2}\right)}, \quad \widetilde{E}_{4}=A_{\left(x_{2}, y_{3}\right)}=\mathcal{X} \times \mathcal{Y} .
$$

We have depicted the focal sets $E_{i}$ and $\widetilde{E}_{i}$ and their connection in Figure 3.
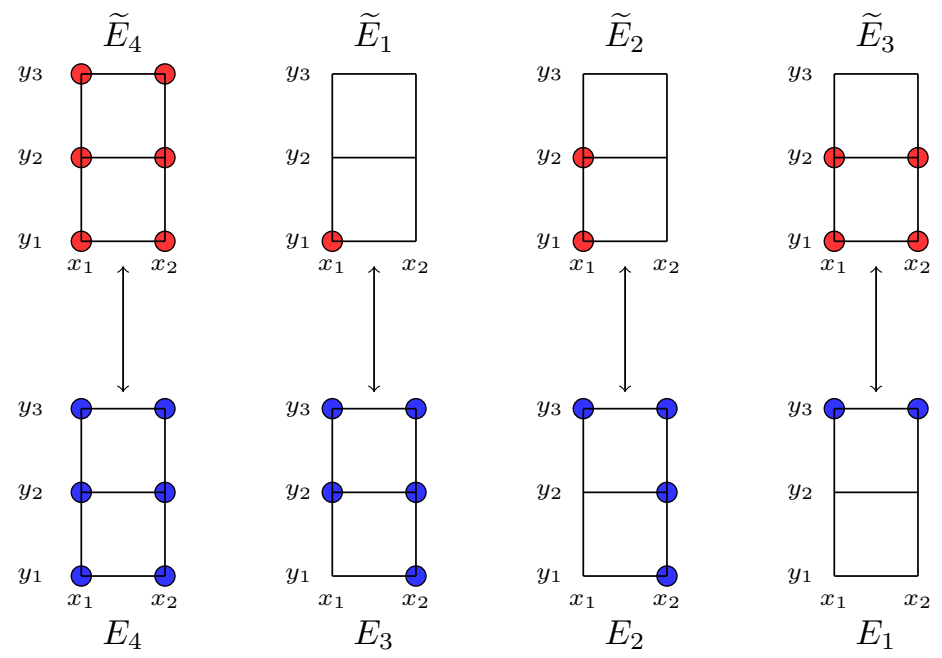

Fig. 3: Connection between type-1 and (Type2-a) possibility measures.

The above connection between type- 1 and type- 2 optimal possibility measures allows us to define the level of the focal set of a type-1 possibility measure. 
Definition 7. Let $\Pi$ be an optimal type-1 possibility with focal sets $E_{1} \subset \ldots \subset$ $E_{n+m-1}$, and denote by $\widetilde{\Pi}$ its associated type- 2 possibility with focal sets $\widetilde{E}_{1} \subset$ $\ldots \subset \widetilde{E}_{n+m-1}$, given by Equation (11). We define the level of $E_{j}$, level $\left(E_{j}\right)$, as:

$$
\operatorname{level}\left(E_{j}\right)=\operatorname{level}\left(\widetilde{E}_{n+m-j}\right) .
$$

As we did with type-2 possibility measures in Section 4.3, we can also give a graphical representation of type- 1 optimal possibility measures. For the case $n=3, m=4$, Figure 4 shows that any optimal type- 1 possibility must move either from $(3,3)$ or $(2,4)$ to $(1,1)$ by following the arrows, where $(i, j)$ represents the focal set $A_{\left(x_{i}, y_{j}\right)}^{c}$, and also taking into account that the last focal set is not depicted because it should be $\mathcal{X} \times \mathcal{Y}$.

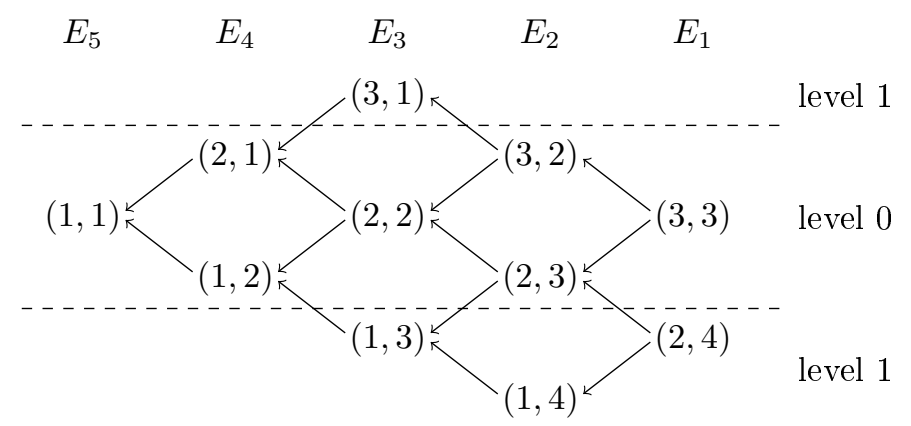

Fig. 4: Optimal type- 1 possibility measures for $n=3, m=4$. The element $(i, j)$ refers to the focal set $A_{\left(x_{i}, y_{j}\right)}^{c}$, and $E_{6}=A_{x_{3}, x_{4}}$.

We are now ready to give the main results, that are the counterparts to Proposition 7 and Theorem 3 .

Proposition 8. Let $\Pi$ and $\Pi^{\prime}$ be two optimal type-1 possibility measures with focal sets $E_{1} \subset \ldots \subset E_{n+m-1}$ and $E_{1}^{\prime} \subset \ldots \subset E_{n+m-1}^{\prime}$, respectively. If level $\left(E_{j}\right) \leq$ level $\left(E_{j}^{\prime}\right) \forall j$, with strict inequality in some $j>1$, then $\mathcal{M}(\Pi)$ has more extreme points than $\mathcal{M}\left(\Pi^{\prime}\right)$. When the inequality is only strict for $j=1$, both $\mathcal{M}(\Pi)$ and $\mathcal{M}\left(\Pi^{\prime}\right)$ have the same number of extreme points.

This result allows us to compute the maximal number of extreme points generated by a type- 1 possibility measure.

Theorem 4. Let $\Pi$ be an optimal type-1 possibility measure on $\mathcal{P}(\mathcal{X} \times \mathcal{Y})$, and assume without loss of generality that $n \leq m$ (otherwise we just need to switch the role of $n$ and $m$ ). The maximal number of extreme points of $\mathcal{M}(\Pi)$ is:

$$
(n !)^{2} n(n+1)^{m-n} .
$$


When $n=m$ or $n<m-1$, this maximum is attained if and only if level $\left(E_{j}\right)=0$ for any $j=m-n, \ldots, m+n-1$. When $n=m-1$, this maximum is also attained when level $\left(E_{j}\right)=0$ for $j>1$ and level $\left(E_{1}\right)=1$.

The minimal number of extreme points of $\mathcal{M}(\Pi)$ is given by $m 2^{m}(m+1)^{n-2}$.

The following example illustrates the result.

Example 5. Consider $\mathcal{X}=\left\{x_{1}, x_{2}, x_{3}\right\}$ and $\mathcal{Y}=\left\{y_{1}, y_{2}, y_{3}, y_{4}\right\}$, that is, $n=3$ and $m=4$. In Figure 5 we have depicted all the optimal type- 1 possibility measures. The ones attaining the maximal number of extreme points are those whose focal sets have zero levels, as well as the type-1 possibilities whose focal sets are:

$$
E_{1}=A_{\left(x_{2}, y_{4}\right)}^{c}, \quad E_{2}=A_{\left(x_{2}, y_{3}\right)}^{c}, \quad E_{3}=A_{\left(x_{2}, y_{2}\right)}^{c}, \quad E_{5}=A_{\left(x_{1}, y_{1}\right)}^{c}, \quad E_{6}=\mathcal{X} \times \mathcal{Y},
$$

and where $E_{4}$ is either $A_{\left(x_{2}, y_{1}\right)}^{c}$ or $A_{\left(x_{1}, y_{2}\right)}^{c}$. Furthermore, the maximum number of extreme points is:

$$
(n !)^{2} n(n+1)^{m-n}=(3 !)^{2} \cdot 3 \cdot 4^{1}=36 \cdot 12=432,
$$

and the minimum one is $m 2^{m}(m+1)^{n-2}=320$. If we compare these with the maximum and minimum number of extreme points of optimal type- 2 possibility measures, established in Theorem 3, we obtain that the maximal number is

$$
\frac{(n !)^{2}(n+1)^{m-n+1}}{2}=288,
$$

while the minimal number is $2^{m-1}(m+1)^{n-1}=200$.

Taking into account Theorems 3 and 4, we conclude that given a maxitive bivariate $p$-box on $\mathcal{X} \times \mathcal{Y}$, where $|\mathcal{X}|=n \leq|\mathcal{Y}|=m$, the maximal number of extreme points is attained with the optimal type- 1 possibility measures.

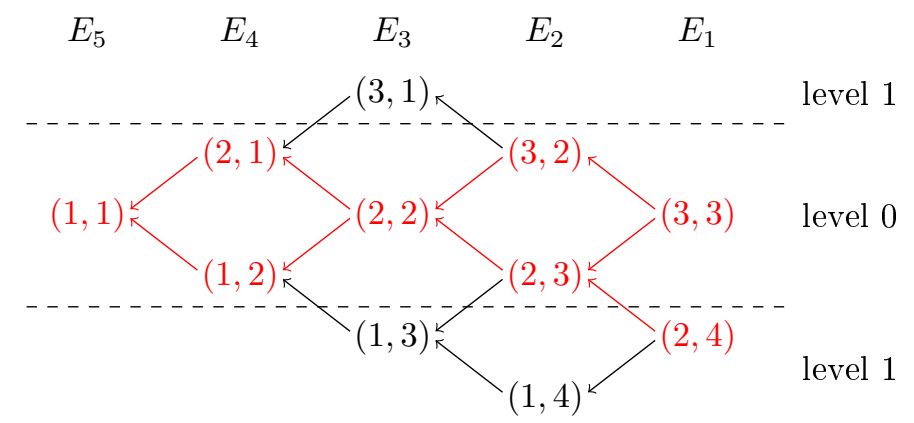

Fig. 5: Optimal type- 1 possibility measures for $n=3, m=4$. The ones whose core has the maximal number of extreme points are those whose focal sets go from either $(3,3)$ or $(2,4)$ to $(1,1)$ through red nodes and arrows. 


\section{Conclusions}

Under an epistemic interpretation, an imprecise probability model can be regarded as equivalent to a set of compatible probability measures. This set is closed and convex, and it is uniquely determined by its extreme points. The number and features of these extreme points are thus important for a number of practical purposes, for instance when dealing with credal networks.

The following table summarizes the maximum number of extreme points for the core of the different imprecise probability models in the univariate case, in terms of the cardinality $n$ of the possibility space:

\begin{tabular}{c|c}
$\mu$ & $|\operatorname{ext}(\mathcal{M}(\mu))|$ \\
\hline Coherent upper probability & $n !^{28}$ \\
2-alternating capacity & $n !^{24}$ \\
Plausibility function & $n !^{4}$ \\
Possibility measure & $2^{n-1} 14$ \\
Univariate $p$-box & $\mathcal{P}_{n}^{16}$ \\
Maxitive univariate $p$-box & $2^{n-1}$ \\
Comparative probabilities & $2^{n-1} 15$
\end{tabular}

In this paper, based on the formula established in ${ }^{22}$ for the number of extreme points of the core of a possibility measure, we have investigated the number of extreme points of the core of maxitive uni- and bivariate probability boxes. The maximum number of extreme points is in those cases noticeably inferior to the case of arbitrary coherent upper probabilities, or that of plausibility functions. Moreover, we have determined in which cases the bound is attained. Interestingly, the formula from ${ }^{22}$ shows that the number of extreme points depends on the number and cardinality of the focal sets of the possibility measure, but not on their masses.

One additional difficulty in the bivariate case is that, unlike the univariate case, the upper probability associated with a bivariate $p$-box need not be a plausibility function, or even coherent; for this reason, we have focused on one particular subfamily of bivariate $p$-boxes: those whose associated upper probability is maxitive. For those, the maximum number of extreme points of the core is given by:

\begin{tabular}{c|c}
$(\underline{F}, \bar{F})$ & $|\operatorname{ext}(\mathcal{M}(\underline{F}, \bar{F}))|$ \\
\hline Type-2 maxitive & $\frac{(n !)^{2}(n+1)^{m-n+1}}{2}$ \\
Type-1 maxitive & $(n !)^{2} n(n+1)^{m-n}$
\end{tabular}

In these formulas, we assume $n=|\mathcal{X}| \leq m=|\mathcal{Y}|$; they can be given for the general case in terms of $\max \{n, m\}$ and $\min \{n, m\}$. Notice how the maximum number of extreme points is different for the two types of bivariate $p$-boxes that may induce a possibility measure: the number for type- 2 -boxes is $\frac{n+1}{2 n}$ times the number for type- 1 bivariate $p$-boxes. This lack os symmetry was already present in the results in ${ }^{17}$, and it is due to the use of different families of cumulative rectangles.

In any case, we see that the maximum number of extreme points is much smaller 
than that of an arbitrary possibility measure on $\mathcal{P}(\mathcal{X} \times \mathcal{Y})$, which is $2^{n m-1}$ : this is because not every possibility measure can be obtained as the upper probability of a bivariate $p$-box, and it also means that maxitive bivariate $p$-boxes should be more tractable from a computational point of view, because as we have seen, for maxitive bivariate $p$-boxes either $\underline{F}$ or $\bar{F}$ is vacuous. One side remark here is that in our results we are employing the assumption made in ${ }^{17}$ that every pair $\left(x_{i}, y_{j}\right)$ has strictly positive upper probability: nevertheless, for maxitive bivariate $p$-boxes this simply means that the union of the focal sets must be equal to the product $\mathcal{X} \times \mathcal{Y}$.

More generally, it would be interesting to investigate the maximum number of extreme points for the core of bivariate $p$-boxes that induce a plausibility function or a coherent upper probability, but that are not maxitive. In this sense, we should first of all characterize those bivariate $p$-boxes that are coherent (some results in this sense can be found in ${ }^{21}$ ) and those that induce a plausibility function. We think that a multi-valued mapping representation might be useful for this second problem.

\section{Acknowledgements}

The research reported in this paper has been supported by project TIN2014-59543P. We would like to thank the reviewers for their useful comments.

\section{References}

1. A. Chateauneuf and J.-Y. Jaffray. Some characterizations of lower probabilities and other monotone capacities through the use of Möbius inversion. Mathematical Social Sciences, 17(3):263-283, 1989.

2. G. Choquet. Theory of capacities. Annales de l'Institut Fourier, 5:131-295, 1953-1954.

3. F. Cozman. Graphical models for imprecise probabilities. International Journal of Approximate Reasoning, 39(2-3):167-184, 2005.

4. A. P. Dempster. Upper and lower probabilities induced by a multivalued mapping. Annals of Mathematical Statistics, 38:325-339, 1967.

5. J. Derks, H. Haller, and H. Peters. The selectope for cooperative games. International Journal of Game Theory, 29:23-38, 2000.

6. S. Destercke, D. Dubois, and E. Chojnacki. Unifying practical uncertainty representations: I. Generalized p-boxes. International Journal of Approximate Reasoning, 49(3):649-663, 2008.

7. D. Dubois and H. Prade. Possibility Theory. Plenum Press, New York, 1988.

8. R. Fagin and J. Y. Halpern. A new approach to updating beliefs. In P. P. Bonissone, M. Henrion, L. N. Kanal, and J. F. Lemmer, editors, Uncertainty in Artificial Intelligence, volume 6, pages 347-374. North-Holland, Amsterdam, 1991.

9. S. Ferson, V. Kreinovich, L. Ginzburg, D. S. Myers, and K. Sentz. Constructing probability boxes and Dempster-Shafer structures. Technical Report SAND2002-4015, Sandia National Laboratories, January 2003.

10. E. Kriegler. Utilizing belief functions for the estimation of future climate change. International Journal of Approximate Reasoning, 39(2-3):185-209, 2005.

11. T. Kroupa. Geometry of cores of submodular coherent upper probabilities and possibility measures. In Soft Methods for Handling Variability and Imprecision, pages 306-312. Springer, 2008. 
12. T. Kroupa. Geometry of possibility measures on finite sets. International Journal of Approximate Reasoning, 48:237-245, 2008.

13. E. Miranda, I. Couso, and P. Gil. Relationships between possibility measures and nested random sets. International Journal of Uncertainty, Fuzziness and KnowledgeBased Systems, 10(1):1-15, 2002.

14. E. Miranda, I. Couso, and P. Gil. Extreme points of credal sets generated by 2alternating capacities. International Journal of Approximate Reasoning, 33(1):95-115, 2003.

15. E. Miranda and S. Destercke. Extreme points of the credal sets generated by comparative probabilities. Journal of Mathematical Psychology, 64/65:44-57, 2015.

16. I. Montes and S. Destercke. Extreme points of p-boxes and belief functions. Annals of Mathematics and Artificial Intelligence, 81(3):405-428, 2017.

17. I. Montes and E. Miranda. Bivariate p-boxes and maxitive functions. International Journal of General Systems, 46(4):354-385, 2017.

18. I. Montes, E. Miranda, R. Pelessoni, and P. Vicig. Sklar's theorem in an imprecise setting. Fuzzy Sets and Systems, 278C:48-66, 2015.

19. H. T. Nguyen. On random sets and belief functions. Journal of Mathematical Analysis and Applications, 65(3):531-542, 1978.

20. H. T. Nguyen. Some mathematical structures for computational information. Information Sciences, 128(1-2):67-89, 2000.

21. R. Pelessoni, P. Vicig, I. Montes, and E. Miranda. Bivariate p-boxes. International Journal of Uncertainty, Fuzziness and Knowledge-Based Systems, 24(2):229-263, 2016.

22. G. Schollmeyer. On the number and characterization of the extreme points of the core of necessity measures on finite spaces. In T. Augustin, S. Doria, E. Miranda, and E. Quaeghebeur, editors, Proceedings of the Ninth International Symposium on Imprecise Probability: Theories and Applications (ISIPTA'2015), pages 277-286, 2015.

23. G. Shafer. A Mathematical Theory of Evidence. Princeton University Press, Princeton, NJ, 1976.

24. L. S. Shapley. Cores of convex games. International Journal of Game Theory, 1:11-26, 1971.

25. M. C. M. Troffaes and S. Destercke. Probability boxes on totally preordered spaces for multivariate modelling. International Journal of Approximate Reasoning, 52(6):767791, 2011.

26. M. C. M. Troffaes, E. Miranda, and S. Destercke. On the connection between probability boxes and possibility measures. Information Sciences, 224:88-108, 2013.

27. P. Walley. Statistical Reasoning with Imprecise Probabilities. Chapman and Hall, London, 1991.

28. A. Wallner. Extreme points of coherent probabilities in finite spaces. International Journal of Approximate Reasoning, 44(3):339-357, 2007.

29. L. A. Zadeh. Fuzzy sets as a basis for a theory of possibility. Fuzzy Sets and Systems, $1: 3-28,1978$.

\section{Appendix. Proofs}

Proof. [Proof of Proposition 1] Using Theorem 1, $\mathcal{M}(\Pi)$ has $k_{1}\left(1+k_{2}\right) \cdot \ldots \cdot\left(1+k_{l}\right)$ extreme points, where the values $k_{i}$ are given in Equation (3). For $\Pi^{\prime}$, we have the following values:

$$
k_{j}^{\prime}=k_{j} \text { for any } j \neq i+1, \quad k_{i+1,1}^{\prime}=\left|E^{*} \backslash E_{i}\right|, \quad k_{i+1,2}^{\prime}=\left|E_{i+1} \backslash E^{*}\right|,
$$


where obviously $k_{i+1,1}^{\prime}+k_{i+1,2}^{\prime}=k_{i+1}$. This implies that:

$$
\begin{aligned}
\left(1+k_{i+1,1}^{\prime}\right)\left(1+k_{i+1,2}^{\prime}\right) & =1+k_{i+1,1}^{\prime} k_{i+1,2}^{\prime}+k_{i+1,1}^{\prime}+k_{i+1,2}^{\prime} \\
& =\left(1+k_{i+1}\right)+k_{i+1,1}^{\prime} k_{i+1,2}^{\prime}>1+k_{i+1} .
\end{aligned}
$$

Therefore, the number of extreme points of $\mathcal{M}\left(\Pi^{\prime}\right)$ is:

$$
\begin{gathered}
k_{1}^{\prime}\left(1+k_{2}^{\prime}\right) \cdot \ldots \cdot\left(1+k_{i}^{\prime}\right) \cdot\left(1+k_{i+1,1}^{\prime}\right)\left(1+k_{i+1,2}^{\prime}\right)\left(1+k_{i+2}^{\prime}\right) \cdot \ldots \cdot\left(1+k_{l}^{\prime}\right) \\
>k_{1}\left(1+k_{2}\right) \cdot \ldots \cdot\left(1+k_{i}\right) \cdot\left(1+k_{i+1}\right) \cdot\left(1+k_{i+2}\right) \cdot \ldots \cdot\left(1+k_{l}\right) .
\end{gathered}
$$

Proof. [Proof of Proposition 2] By Theorem 1, the number of extreme points of $\mathcal{M}(\Pi)$ is $k_{1}\left(1+k_{2}\right) \cdot \ldots \cdot\left(1+k_{l}\right)$, where again the values $k_{i}$ are given in Equation (3). On the other hand, in the case of $\mathcal{M}\left(\Pi^{\prime}\right)$, Equation (3) gives:

$$
k=\left|E^{*}\right|, k^{\prime}=\left|E_{1} \backslash E^{*}\right|, k_{i}^{\prime}=\left|E_{i} \backslash E_{i-1}\right|=k_{i} \forall i=2, \ldots, l,
$$

where $k+k^{\prime}=k_{1}$, and therefore:

$$
k\left(1+k^{\prime}\right)=k+k k^{\prime} \geq k+k^{\prime}=k_{1} ;
$$

the inequality is strict when $k>1$. Thus, the number of extreme points of $\mathcal{M}\left(\Pi^{\prime}\right)$ is given by:

$$
k\left(1+k^{\prime}\right)\left(1+k_{2}\right) \cdot \ldots \cdot\left(1+k_{l}\right) \geq k_{1}\left(1+k_{2}\right) \cdot \ldots \cdot\left(1+k_{l}\right)=|\operatorname{ext}(\mathcal{M}(\Pi))|,
$$

where the last inequality is strict when $\left|E^{*}\right|=k>1$.

Proof. [Proof of Corollary 1] That both these conditions are sufficient for $\mathcal{M}(\Pi)$ to have $2^{n-1}$ extreme points follows from Theorem 1.

To see that they are also necessary, assume ex-absurdo that there is some $i \in$ $\{2, \ldots, l\}$ with $k_{i}>1$. Then, there we can find a set $E^{*}$ such that $E_{i-1} \subset E^{*} \subset E_{i}$, and applying Proposition 1 we can use it to determine a possibility measure $\Pi^{\prime}$ such that $\mathcal{M}\left(\Pi^{\prime}\right)$ has more extreme points that $\mathcal{M}(\Pi)$. Thus, $\mathcal{M}(\Pi)$ does not have the maximal number of extreme points.

Proof. [Proof of Proposition 4] Let $E_{1} \subset \ldots \subset E_{l}$ be the focal sets of $\Pi$, where $E_{l}=A_{\left(x_{n}, y_{m}\right)}=\mathcal{X} \times \mathcal{Y}$. By Corollary 1 , in order for $\mathcal{M}(\Pi)$ to have $2^{n m-1}$ extreme points, the set $E_{l} \backslash E_{l-1}$ should have one element, i.e., $E_{l-1}=\mathcal{X} \times \mathcal{Y} \backslash\left\{\left(x_{n}, y_{m}\right)\right\}$. However, when $n, m>1$ this set is not a cumulative rectangle. Thus, $\mathcal{M}(\Pi)$ cannot have $2^{n m-1}$ extreme points.

Proof. [Proof of Proposition 5]

(i) Let us prove that level $\left(E_{2 j+1}\right) \geq \operatorname{level}\left(E_{2 j+2}\right)$ for every $j$.

Assume that $E_{2 j+1}=A_{\left(x_{r}, y_{s}\right)}$. Since $r+s$ is even by Equation (9), we have that level $\left(E_{2 j+1}\right)=\frac{|r-s|}{2}$, because $r+s,|r-s|$ have the same parity. Taking conditions $(\mathrm{C} 1),(\mathrm{C} 2)$ into account, there are two possibilities: either $E_{2 j+2}=A_{\left(x_{r+1}, y_{s}\right)}$, whence level $\left(E_{2 j+2}\right)=\left\lfloor\frac{|r+1-s|}{2}\right\rfloor$; or $E_{2 j+2}=A_{\left(x_{r}, y_{s+1}\right)}$, whence level $\left(E_{2 j+2}\right)=\left\lfloor\frac{|r-s-1|}{2}\right\rfloor$. 
In any case, $|r+1-s|$ and $|r-s-1|$ cannot be greater than $|r-s|+1$, so level $\left(E_{2 j+2}\right) \leq \frac{|r-s|}{2}=\operatorname{level}\left(E_{2 j+1}\right)$.

In a similar manner we can show that level $\left(E_{2 j+1}\right) \geq \operatorname{level}\left(E_{2 j}\right)$ for every $j$ : conditions $(\mathrm{C} 1)$ and $(\mathrm{C} 2)$ imply that either $E_{2 j}=A_{\left(x_{r-1}, y_{s}\right)}$, whence level $\left(E_{2 j}\right)=\left\lfloor\frac{|r-1-s|}{2}\right\rfloor$; or $E_{2 j}=A_{\left(x_{r}, y_{s-1}\right)}$, whence level $\left(E_{2 j+2}\right)=$ $\left\lfloor\frac{|r-s+1|}{2}\right\rfloor$. Again $|r-1-s|$ and $|r-s+1|$ cannot be greater than $|r-s|+1$, whence level $\left(E_{2 j}\right) \leq \frac{|r-s|}{2}=\operatorname{level}\left(E_{2 j+1}\right)$.

(ii) Let us prove next the second statement; the proof of the third is analogous. We already know from the first statement that level $\left(E_{2 j}\right) \leq \operatorname{level}\left(E_{2 j+1}\right)$. Moreover, if $E_{2 j}=A_{\left(x_{r}, x_{s}\right)}$, then level $\left(E_{2 j}\right)=\frac{|r-s-1|}{2}$, because $|r-s|$ is odd. Since either $E_{2 j+1}=A_{\left(x_{r+1}, y_{s}\right)}$ or $E_{2 j+1}=A_{\left(x_{r}, y_{s+1}\right)}$, we have the following two possibilities:

a) If level $\left(E_{2 j+1}\right)=\operatorname{level}\left(E_{2 j}\right)$, then $E_{2 j+1}=A_{\left(x_{r+1}, y_{s}\right)}$, and therefore:

$$
k_{2 j+1}=\left|E_{2 j+1} \backslash E_{2 j}\right|=(r+1) \cdot s-r \cdot s=s .
$$

b) If level $\left(E_{2 j+1}\right)=\operatorname{level}\left(E_{2 j}\right)+1$, then $E_{2 j+1}=A_{\left(x_{r}, y_{s+1}\right)}$, and therefore:

$$
k_{2 j+1}=\left|E_{2 j+1} \backslash E_{2 j}\right|=(s+1) \cdot r-s \cdot r=r .
$$

Proof. [Proof of Proposition 6] We begin with the first statement. Let $E_{i}=$ $A_{\left(x_{r_{i}}, y_{s_{i}}\right)}$ and without loss of generality assume that $r_{i} \leq s_{i}$. Take $E_{i}^{\prime}=A_{\left(x_{r_{i}^{\prime}}, y_{s_{i}^{\prime}}\right)}$ and assume that level $\left(E_{i}\right)=\operatorname{level}\left(E_{i}^{\prime}\right)$. By Equation (9), we know that $r_{i}+s_{i}=$ $i+1=r_{i}^{\prime}+s_{i}^{\prime}$. Consider two cases:

Case $1 i$ is odd. Then:

$$
\frac{\left|s_{i}-r_{i}\right|}{2}=\operatorname{level}\left(E_{i}\right)=\operatorname{level}\left(E_{i}^{\prime}\right)=\frac{\left|r_{i}^{\prime}-s_{i}^{\prime}\right|}{2} .
$$

This implies that $s_{i}-r_{i}=\left|s_{i}^{\prime}-r_{i}^{\prime}\right|$, whence either $E_{i}^{\prime}=A_{\left(x_{r_{i}}, y_{s_{i}}\right)}$ or $E_{i}^{\prime}=A_{\left(x_{s_{i}}, y_{r_{i}}\right)}$.

Case $2 i$ is even. Then:

$$
\frac{\left|s_{i}-r_{i}\right|-1}{2}=\operatorname{level}\left(E_{i}\right)=\operatorname{level}\left(E_{i}^{\prime}\right)=\frac{\left|r_{i}^{\prime}-s_{i}^{\prime}\right|-1}{2} .
$$

Thus, $s_{i}-r_{i}=\left|s_{i}^{\prime}-r_{i}^{\prime}\right|$, and as a consequence either $E_{i}^{\prime}=A_{\left(x_{r_{i}}, y_{s_{i}}\right)}$ or $E_{i}^{\prime}=A_{\left(x_{s_{i}}, y_{r_{i}}\right)}$.

From this we deduce that $\min \left\{r_{i}, s_{i}\right\}=\min \left\{r_{i}^{\prime}, s_{i}^{\prime}\right\}$ and $\max \left\{r_{i}, s_{i}\right\}=\max \left\{r_{i}^{\prime}, s_{i}^{\prime}\right\}$.

We turn now towards the second statement.

Let us prove that $E_{i}^{\prime \prime} \subset E_{i+1}^{\prime \prime}$ for any $i=1, \ldots, n+m-2$. We have the following possible scenarios:

(a) $E_{i}^{\prime \prime}=E_{i}$ and $E_{i+1}^{\prime \prime}=E_{i+1}$. Then trivially $E_{i}^{\prime \prime} \subset E_{i+1}^{\prime \prime}$ because $E_{i} \subset E_{i+1}$.

(b) $E_{i}^{\prime \prime}=E_{i}$ and $E_{i+1}^{\prime \prime} \neq E_{i+1}$. This means that:

$$
E_{i}=A_{\left(x_{r_{i}}, y_{r_{i}}\right)}=E_{i}^{\prime \prime}, \quad E_{i+1}=A_{\left(x_{r_{i}+1}, y_{r_{i}}\right)} \text { and } E_{i+1}^{\prime \prime}=A_{\left(x_{r_{i}}, y_{r_{i}+1}\right)},
$$


whence $E_{i}^{\prime \prime} \subset E_{i+1}^{\prime \prime}$.

(c) $E_{i}^{\prime \prime} \neq E_{i}$ and $E_{i+1}^{\prime \prime}=E_{i+1}$. This means that:

$$
E_{i}=A_{\left(x_{r_{i}}, y_{r_{i}-1}\right)}, \quad E_{i}^{\prime \prime}=A_{\left(x_{r_{i}-1}, y_{r_{i}}\right)}, \quad E_{i+1}=A_{\left(x_{r_{i}}, y_{r_{i}}\right)}=E_{i+1}^{\prime \prime} .
$$

Thus, $E_{i}^{\prime \prime} \subset E_{i+1}^{\prime \prime}$.

(d) $E_{i}^{\prime \prime} \neq E_{i}$ and $E_{i+1}^{\prime \prime} \neq E_{i+1}$. This means that $E_{i}=A_{\left(x_{r_{i}}, y_{s_{i}}\right)}$ and $E_{i+1}=$ $A_{\left(x_{r_{i+1}}, y_{s_{i+1}}\right)}$ with $r_{i}>s_{i}$ and $r_{i+1}>s_{i+1}$ with $r_{i} \leq r_{i+1}, s_{i} \leq s_{i+1}$. Then:

$$
E_{i}^{\prime \prime}=A_{\left(x_{s_{i}}, y_{r_{i}}\right)} \text { and } E_{i+1}=A_{\left(x_{s_{i+1}}, y_{r_{i+1}}\right)},
$$

and therefore $E_{i}^{\prime \prime} \subset E_{i+1}^{\prime \prime}$.

Now, by definition it is clear that both $E_{i}$ and $E_{i}^{\prime \prime}$ have the same level $\left(\left|r_{i}-s_{i}\right|=\right.$ $\left.\left|s_{i}-r_{i}\right|\right)$. Applying Proposition 5 we deduce that the values $k_{i}$ and $k_{i}^{\prime \prime}$ also coincide and consequently using Equation (4), $|\operatorname{ext}(\mathcal{M}(\Pi))|=\left|\operatorname{ext}\left(\mathcal{M}\left(\Pi^{\prime \prime}\right)\right)\right|$.

In order to prove Proposition 7, we must establish first a number of auxiliary results:

Lemma 1. Let $\Pi, \Pi^{\prime}$ be two optimal type-2 possibility measures. Assume that they satisfy the following properties:

(1) The focal sets of $\Pi$ are given by $E_{1} \subset \ldots \subset E_{n+m-1}$ such that $E_{i}=A_{\left(x_{r}, y_{s}\right)}$, $E_{i+1}=A_{\left(x_{r+1}, y_{s}\right)}$ and $E_{i+2}=A_{\left(x_{r+1}, y_{s+1}\right)}$.

(2) The focal sets of $\Pi^{\prime}$ are given by

$$
E_{1} \subset \ldots \subset E_{i} \subset E_{i+1}^{\prime} \subset E_{i+2} \subset \ldots \subset E_{n+m-1},
$$

(3) $s>r$.

$$
\text { with } E_{i}=A_{\left(x_{r}, y_{s}\right)}, E_{i+1}=A_{\left(x_{r}, y_{s+1}\right)} \text { and } E_{i+2}=A_{\left(x_{r+1}, y_{s+1}\right)} \text {. }
$$

Then, $|\operatorname{ext}(\mathcal{M}(\Pi))|>\left|\operatorname{ext}\left(\mathcal{M}\left(\Pi^{\prime}\right)\right)\right|$.

Proof. From Equation (4), we only need to show that $\left(1+k_{i+1}\right)\left(1+k_{i+2}\right)>$ $\left(1+k_{i+1}^{\prime}\right)\left(1+k_{i+2}^{\prime}\right)$, where $k_{j}=\left|E_{j} \backslash E_{j-1}\right|$ and $k_{j}^{\prime}=\left|E_{j}^{\prime} \backslash E_{j-1}^{\prime}\right|$. From the hypotheses, we obtain

$$
\begin{aligned}
& k_{i+1}=\left|E_{i+1} \backslash E_{i}\right|=(r+1) \cdot s-r \cdot s=s \\
& k_{i+2}=\left|E_{i+2} \backslash E_{i+1}\right|=(r+1) \cdot(s+1)-(r+1) s=r+1
\end{aligned}
$$

while

$$
\begin{aligned}
& k_{i+1}^{\prime}=\left|E_{i+1} \backslash E_{i}\right|=(s+1) \cdot r-s \cdot r=r . \\
& k_{i+2}^{\prime}=\left|E_{i+2} \backslash E_{i+1}\right|=(r+1) \cdot(s+1)-(s+1) \cdot r=s+1 .
\end{aligned}
$$

Now, since $s>r$,

$$
\left(k_{i+1}+1\right) \cdot\left(k_{i+2}+1\right)=(s+1)(r+2)>(r+1)(s+2)=\left(k_{i+1}^{\prime}+1\right) \cdot\left(k_{i+2}^{\prime}+1\right),
$$

and as a consequence $\mathcal{M}(\Pi)$ has more extreme points than $\mathcal{M}\left(\Pi^{\prime}\right)$. 
Corollary 2. Let $\Pi, \Pi^{\prime}$ be two optimal type-2 possibility measures. Assume that they satisfy the following properties:

(1) The focal sets of $\Pi$ are given by $E_{1} \subset \ldots \subset E_{n+m-1}$ such that level $\left(E_{i}\right)=$ level $\left(E_{i+2}\right)$.

(2) The focal sets of $\Pi^{\prime}$ are given by

$$
\begin{aligned}
& \qquad E_{1} \subset \ldots \subset E_{i} \subset E_{i+1}^{\prime} \subset E_{i+2} \subset \ldots \subset E_{n+m-1} \\
& \text { such that level }\left(E_{i+1}^{\prime}\right)>\operatorname{level}\left(E_{i+1}\right) .
\end{aligned}
$$

Then, $|\operatorname{ext}(\mathcal{M}(\Pi))|>\left|\operatorname{ext}\left(\mathcal{M}\left(\Pi^{\prime}\right)\right)\right|$.

Proof. This is a straightforward reformulation of Lemma 1 in terms of levels.

Lemma 2. Let $\Pi$ and $\Pi^{\prime}$ be two optimal type-2 possibility measures on $\mathcal{P}(\mathcal{X} \times \mathcal{Y})$, with respective focal sets $E_{1} \subset \ldots \subset E_{n+m-1}$ and $E_{1}^{\prime} \subset \ldots \subset E_{n+m-1}^{\prime}$. Assume that level $\left(E_{j}^{\prime}\right)$-level $\left(E_{j}\right) \in\{0,1\}$ for any $j=2, \ldots, n+m-1$ and that level $\left(E_{j}^{\prime}\right)-\operatorname{level}\left(E_{j}\right)=1$ for at least one $j$. Then, $|\operatorname{ext}(\mathcal{M}(\Pi))|>\left|\operatorname{ext}\left(\mathcal{M}\left(\Pi^{\prime}\right)\right)\right|$.

Proof. Taking into account Proposition 6, we can assume without loss of generality that if $A_{\left(x_{r}, y_{s}\right)}$ is a focal set $E_{i}$ or $E_{i}^{\prime}$, then $r \leq s$.

Let us proceed by induction on

$$
\left.\gamma=\mid j \in\{2, \ldots, n+m-1\}: \operatorname{level}\left(E_{j}^{\prime}\right)-\operatorname{level}\left(E_{j}\right)=1\right\} \mid .
$$

First of all, assume that $\gamma=1$, and let $j+1 \operatorname{satisfy} \operatorname{level}\left(E_{j+1}^{\prime}\right)-\operatorname{level}\left(E_{j+1}\right)=1$. Thus, level $\left(E_{i}\right)=\operatorname{level}\left(E_{i}^{\prime}\right)$ for any $i \neq j+1$ because $\gamma=1$, and therefore, according to Proposition $6, E_{i}=E_{i}^{\prime}$ for any $i \neq j+1$. There are two possible cases:

(1) $i=\operatorname{level}\left(E_{j}\right)=\operatorname{level}\left(E_{j}^{\prime}\right)=\operatorname{level}\left(E_{j+1}^{\prime}\right)>i-1=\operatorname{level}\left(E_{j+1}\right)$. According to Proposition 5, $j$ must be odd. Also, since $\gamma=1$, conditions (L1)-(L2) imply that level $\left(E_{j+2}\right)=\operatorname{level}\left(E_{j+2}^{\prime}\right)=i-1$. Since $E_{i}=E_{i}^{\prime}$ for any $i \neq j+1$, Corollary 2 implies that $\mathcal{M}(\Pi)$ has more extreme points than $\mathcal{M}\left(\Pi^{\prime}\right)$.

(2) $i=\operatorname{level}\left(E_{j}\right)=\operatorname{level}\left(E_{j}^{\prime}\right)=\operatorname{level}\left(E_{j+1}\right)<i+1=\operatorname{level}\left(E_{j+1}^{\prime}\right)$. By Proposition $5, j$ must be even. Also, since $\gamma=1$, the same result tells us that level $\left(E_{j+2}\right)=\operatorname{level}\left(E_{j+2}^{\prime}\right)=i$. Taking into account that $E_{i}=E_{i}^{\prime}$ for any $i \neq j+1$, Corollary 2 implies that $\mathcal{M}(\Pi)$ has more extreme points than $\mathcal{M}\left(\Pi^{\prime}\right)$.

Next we consider the case of $\gamma>1$ and denote $j+1=\min \{2, \ldots, n+m-1$ | level $\left.\left(E_{j}^{\prime}\right)-\operatorname{level}\left(E_{j}\right)=1\right\}$; the idea of the proof is to build another possibility measure $\Pi^{*}$ such that $\mathcal{M}(\Pi)$ has more extreme points than $\mathcal{M}\left(\Pi^{*}\right)$, which in turn has more extreme points such than $\mathcal{M}\left(\Pi^{\prime}\right)$, and to apply the induction hypothesis on $\Pi^{*}$. There are two possible scenarios:

(1) $i=\operatorname{level}\left(E_{j}\right)=\operatorname{level}\left(E_{j}^{\prime}\right)=\operatorname{level}\left(E_{j+1}\right)<\operatorname{level}\left(E_{j+1}^{\prime}\right)=i+1$. According to Proposition 5, $j$ is even. We consider again the following cases: 
(1a) level $\left(E_{j+2}\right)=i<i+1=\operatorname{level}\left(E_{j+2}^{\prime}\right)$. We consider the possibility measure $\Pi^{*}$ whose focal sets are $E_{1}^{*} \subset \ldots \subset E_{n+m-1}^{*}$, where

$$
E_{j+1}^{*}=E_{j+1}^{\prime} \text { and } E_{s}^{*}=E_{s} \text { for any } s \neq j+1 .
$$

Note that the focal sets $E_{i}^{*}$ are nested: obviously $E_{i}^{*} \subset E_{i+1}^{*}$ for any $i=$ $1, \ldots, j-1, j+2, \ldots, n+m-1$ because $E_{i}^{*}=E_{i}$ and $E_{i+1}^{*}=E_{i+1}$. On the other hand, if $E_{j}=A_{\left(x_{r}, y_{s}\right)}$, the equality level $\left(E_{j}^{*}\right)=\operatorname{level}\left(E_{j+2}\right)$ implies that $E_{j+1}^{*}=A_{\left(x_{r+1}, y_{s+1}\right)}$. Therefore, $E_{j+1}^{*}$ is either $A_{\left(x_{r}, y_{s+1}\right)}$ or $A_{\left(x_{r+1}, y_{s}\right)}$, and in both cases $E_{j}^{*} \subset E_{j+1}^{*} \subset E_{j+2}^{*}$.

According to Corollary 2, $\mathcal{M}(\Pi)$ has more extreme points than $\mathcal{M}\left(\Pi^{*}\right)$, level $\left(E_{s}^{*}\right) \leq \operatorname{level}\left(E_{s}^{\prime}\right)$ and

$$
\left|\left\{j \in\{2, \ldots, n+m-1\} \mid \operatorname{level}\left(E_{j}^{\prime}\right)-\operatorname{level}\left(E_{j}^{*}\right)=1\right\}\right|=\gamma-1 .
$$

Applying the induction hypothesis, $\mathcal{M}\left(\Pi^{*}\right)$ has more extreme points than $\mathcal{M}\left(\Pi^{\prime}\right)$, whence $\mathcal{M}(\Pi)$ has more extreme points than $\mathcal{M}\left(\Pi^{\prime}\right)$.

(1b) level $\left(E_{j+2}\right)=i-1<i=\operatorname{level}\left(E_{j+2}^{\prime}\right)$. We consider the possibility measure $\Pi^{*}$ whose focal sets are $E_{1}^{*} \subset \ldots \subset E_{n+m-1}^{*}$, where

$$
E_{j+1}^{*}=E_{j+1} \text { and } E_{s}^{*}=E_{s}^{\prime} \text { for any } s \neq j+1 .
$$

Following the same reasoning than in (1a), we can verify that the sets $E_{i}^{*}$ are nested and that as a consequence $\Pi^{*}$ is a possibility measure. By Corollary 2, $\mathcal{M}\left(\Pi^{*}\right)$ has more extreme points than $\mathcal{M}\left(\Pi^{\prime}\right)$; moreover, $\operatorname{level}\left(E_{s}^{*}\right) \leq \operatorname{level}\left(E_{s}\right)$ and

$$
\left|\left\{j \in\{2, \ldots, n+m-1\} \mid \operatorname{level}\left(E_{j}^{*}\right)-\operatorname{level}\left(E_{j}\right)=1\right\}\right|=\gamma-1 .
$$

Applying the induction hypothesis, $\mathcal{M}(\Pi)$ has more extreme points than $\mathcal{M}\left(\Pi^{*}\right)$, whence $\mathcal{M}(\Pi)$ has more extreme points than $\mathcal{M}\left(\Pi^{\prime}\right)$.

(1c) level $\left(E_{j+2}\right)=i=\operatorname{level}\left(E_{j+2}^{\prime}\right)$. We consider the possibility measure $\Pi^{*}$ whose focal sets are $E_{1}^{*} \subset \ldots \subset E_{n+m-1}^{*}$, where

$$
E_{j+1}^{*}=E_{j+1} \text { and } E_{s}^{*}=E_{s}^{\prime} \text { for any } s \neq j+1 .
$$

Reasoning as in (1a), we can easily verify that the sets $E_{i}^{*}$ are nested and therefore $\Pi^{*}$ is a possibility measure. By Corollary $2, \mathcal{M}\left(\Pi^{*}\right)$ has more extreme points than $\mathcal{M}\left(\Pi^{\prime}\right) ;$ moreover, level $\left(E_{s}\right) \leq \operatorname{level}\left(E_{s}^{*}\right)$ and

$$
\left|\left\{j \in\{2, \ldots, n+m-1\} \mid \operatorname{level}\left(E_{j}^{*}\right)-\operatorname{level}\left(E_{j}\right)=1\right\}\right|=\gamma-1 .
$$

Applying the induction hypothesis, $\mathcal{M}(\Pi)$ has more extreme points than $\mathcal{M}\left(\Pi^{*}\right)$, whence that $\mathcal{M}(\Pi)$ has more extreme points than $\mathcal{M}\left(\Pi^{\prime}\right)$.

(2) The second possible case is that $i=\operatorname{level}\left(E_{j}\right)=\operatorname{level}\left(E_{j}^{\prime}\right)=\operatorname{level}\left(E_{j+1}^{\prime}\right)>$ level $\left(E_{j+1}\right)=i-1$. Here we have three possible scenarios:

(2a) $\operatorname{level}\left(E_{j+2}\right)=i-1<i=\operatorname{level}\left(E_{j+2}^{\prime}\right)$. 
(2b) $\operatorname{level}\left(E_{j+2}\right)=i<i+1=\operatorname{level}\left(E_{j+2}^{\prime}\right)$.

(2c) $\operatorname{level}\left(E_{j+2}\right)=i=\operatorname{level}\left(E_{j+2}^{\prime}\right)$.

The proof of these three cases is analogous to that of (1a)-(1c) above.

Proof. [Proof of Proposition 7] Taking into account Proposition 6, we can assume without loss of generality that if $A_{\left(x_{r}, y_{s}\right)}$ is a focal set of $\Pi$ or $\Pi^{\prime}$, then $r \leq s$.

Consider the value

$$
\alpha=\max \left\{\operatorname{level}\left(E_{j}^{\prime}\right)-\operatorname{level}\left(E_{j}\right): j=2, \ldots, n+m-1\right\} ;
$$

we shall make the proof by induction over $\alpha$. For $\alpha=1$, the result was proven in Lemma 2. Consider the case of $\alpha>1$, and let us define

$$
\gamma=\left|\left\{j \in\{1, \ldots, n+m-1\}: \operatorname{level}\left(E_{j}^{\prime}\right)-\operatorname{level}\left(E_{j}\right)=\alpha\right\}\right| .
$$

Again, let us proceed by induction over $\gamma$. For $\gamma=1$, denote by $j+1$ the unique element such that level $\left(E_{j+1}^{\prime}\right)-\operatorname{level}\left(E_{j+1}\right)=\alpha$. This implies that level $\left(E_{j}^{\prime}\right)-$ level $\left(E_{j}\right)=\alpha-1$. There are two cases:

(1) $i=\operatorname{level}\left(E_{j}\right)>\operatorname{level}\left(E_{j+1}\right)=i-1, i+\alpha-1=\operatorname{level}\left(E_{j}^{\prime}\right)=\operatorname{level}\left(E_{j+1}^{\prime}\right)$. According to Proposition 5, $j$ must be odd. Also, since $\gamma=1$, conditions (L1)-(L2) imply that level $\left(E_{j+2}\right)=i$ and $\operatorname{level}\left(E_{j+2}^{\prime}\right)=i+\alpha-1$. We consider the possibility $\Pi^{*}$ with focal sets $E_{1}^{*} \subset \ldots \subset E_{n+m-1}^{*}$ given by:

$$
E_{j+1}^{*}=E, \quad E_{s}^{*}=E_{s} \text { for any } s \neq j+1,
$$

where $E_{j} \subset E \subset E_{j+2}$ and level $(E)=i$. Then, according to Corollary 2, $\mathcal{M}(\Pi)$ has more extreme points than $\mathcal{M}\left(\Pi^{*}\right)$, level $\left(E_{s}^{*}\right) \leq \operatorname{level}\left(E_{s}^{\prime}\right)$ and

$$
\max \left\{\operatorname{level}\left(E_{j}^{\prime}\right)-\operatorname{level}\left(E_{j}^{*}\right): j=2, \ldots, n+m-1\right\}=\alpha-1
$$

whence by the induction hypothesis, $\mathcal{M}\left(\Pi^{*}\right)$ has more extreme points than $\mathcal{M}\left(\Pi^{\prime}\right)$.

(2) $i=\operatorname{level}\left(E_{j}\right)=\operatorname{level}\left(E_{j+1}\right), i+\alpha-1=\operatorname{level}\left(E_{j}^{\prime}\right)<\operatorname{level}\left(E_{j+1}^{\prime}\right)=i+$ $\alpha$. According to Proposition 5, $j$ must be even. Also, since $\gamma=1$, using conditions (L1)-(L2) we conclude that $\operatorname{level}\left(E_{j+2}\right)=i$ and $\operatorname{level}\left(E_{j+2}^{\prime}\right)=$ $i+\alpha-1$. We consider the possibility $\Pi^{*}$ with focal sets $E_{1}^{*} \subset \ldots \subset E_{n+m-1}^{*}$ given by:

$$
E_{j+1}^{*}=E, \quad E_{s}^{*}=E_{s}^{\prime} \text { for any } s \neq j+1,
$$

where $E_{j}^{\prime} \subset E \subset E_{j+2}^{\prime}$ and level $(E)=i+\alpha-1$. Then, according to Corollary $2, \mathcal{M}\left(\Pi^{*}\right)$ has more extreme points than $\mathcal{M}\left(\Pi^{\prime}\right)$, level $\left(E_{s}\right) \leq$ level $\left(E_{s}^{*}\right)$ and

$$
\max \left\{\operatorname{level}\left(E_{j}^{*}\right)-\operatorname{level}\left(E_{j}\right): j=2, \ldots, n+m-1\right\}=\alpha-1,
$$

whence by induction hypothesis, $\mathcal{M}(\Pi)$ has more extreme points than $\mathcal{M}\left(\Pi^{*}\right)$. 
We conclude in either case that $\mathcal{M}(\Pi)$ has more extreme points than $\mathcal{M}\left(\Pi^{\prime}\right)$.

Next we consider the case of $\gamma>1$ and denote

$$
j+1=\min \left\{2, \ldots, n+m-1: \operatorname{level}\left(E_{j}^{\prime}\right)-\operatorname{level}\left(E_{j}\right)=\alpha\right\} .
$$

This implies that level $\left(E_{j}^{\prime}\right)-\operatorname{level}\left(E_{j}\right)=\alpha-1$. There are two possible cases:

(1) $i=\operatorname{level}\left(E_{j}\right)=\operatorname{level}\left(E_{j+1}\right), i+\alpha-1=\operatorname{level}\left(E_{j}^{\prime}\right)<\operatorname{level}\left(E_{j+1}^{\prime}\right)=i+\alpha$. According to Proposition $5, j$ is even. We consider again the following cases:

(1a) $i=\operatorname{level}\left(E_{j+2}\right), i+\alpha=\operatorname{level}\left(E_{j+2}^{\prime}\right)$. We define the possibility measure $\Pi^{*}$ with focal sets $E_{1}^{*} \subset \ldots \subset E_{n+m-1}^{*}$ given by:

$$
E_{j+1}^{*}=E, \quad E_{s}^{*}=E_{s} \text { for any } s \neq j+1,
$$

where $E_{j} \subset E \subset E_{j+2}$ and level $(E)=i+1$. According to Corollary 2, $\mathcal{M}(\Pi)$ has more extreme points than $\mathcal{M}\left(\Pi^{*}\right), \operatorname{level}\left(E_{s}^{*}\right) \leq \operatorname{level}\left(E_{s}^{\prime}\right)$ for any $s=2, \ldots, n+m-1$ and

$$
\left|\left\{j \in\{2, \ldots, n+m-1\}: \operatorname{level}\left(E_{j}^{\prime}\right)-\operatorname{level}\left(E_{j}^{*}\right)=\alpha\right\}\right|=\gamma-1 .
$$

Applying the induction hypothesis, $\mathcal{M}\left(\Pi^{*}\right)$ has more extreme points than $\mathcal{M}\left(\Pi^{\prime}\right)$, whence $\mathcal{M}(\Pi)$ has more extreme points than $\mathcal{M}\left(\Pi^{\prime}\right)$.

(1b) $i-1=\operatorname{level}\left(E_{j+2}\right), i+\alpha-1=\operatorname{level}\left(E_{j+2}^{\prime}\right)$. We define the possibility measure $\Pi^{*}$ with focal sets $E_{1}^{*} \subset \ldots \subset E_{n+m-1}^{*}$ given by:

$$
E_{j+1}^{*}=E, \quad E_{s}^{*}=E_{s}^{\prime} \text { for any } s \neq j+1,
$$

where $E_{j}^{\prime} \subset E \subset E_{j+2}^{\prime}$ and level $(E)=i+\alpha-1$. According to Corollary $2, \mathcal{M}\left(\Pi^{*}\right)$ has more extreme points than $\mathcal{M}\left(\Pi^{\prime}\right)$, level $\left(E_{s}\right) \leq$ $\operatorname{level}\left(E_{s}^{*}\right)$ for any $s=2, \ldots, n+m-1$ and

$$
\left|\left\{j \in\{2, \ldots, n+m-1\}: \operatorname{level}\left(E_{j}^{*}\right)-\operatorname{level}\left(E_{j}\right)=\alpha\right\}\right|=\gamma-1 .
$$

Applying the induction hypothesis, $\mathcal{M}(\Pi)$ has more extreme points than $\mathcal{M}\left(\Pi^{*}\right)$, whence $\mathcal{M}(\Pi)$ has more extreme points than $\mathcal{M}\left(\Pi^{\prime}\right)$.

(1c) $i=\operatorname{level}\left(E_{j+2}\right), i+\alpha-1=\operatorname{level}\left(E_{j+2}^{\prime}\right)$. We define the possibility measure $\Pi^{*}$ with focal sets $E_{1}^{*} \subset \ldots \subset E_{n+m-1}^{*}$ given by:

$$
E_{j+1}^{*}=E, \quad E_{s}^{*}=E_{s}^{\prime} \text { for any } s \neq j+1,
$$

where $E_{j}^{\prime} \subset E \subset E_{j+2}^{\prime}$ and level $(E)=i+\alpha-1$. By Corollary 2, $\mathcal{M}\left(\Pi^{*}\right)$ has more extreme points than $\mathcal{M}\left(\Pi^{\prime}\right)$, level $\left(E_{s}^{*}\right) \geq \operatorname{level}\left(E_{s}\right)$ for any $s=2, \ldots, n+m-1$ and

$$
\left|\left\{j \in\{2, \ldots, n+m-1\}: \operatorname{level}\left(E_{j}^{\prime}\right)-\operatorname{level}\left(E_{j}\right)=\alpha\right\}\right|=\gamma-1 .
$$

Applying the induction hypothesis, $\mathcal{M}(\Pi)$ has more extreme points than $\mathcal{M}\left(\Pi^{*}\right)$, and as a consequence it also has more extreme points than $\mathcal{M}\left(\Pi^{\prime}\right)$. 
(2) The second possible case is that of $i=\operatorname{level}\left(E_{j}\right)>\operatorname{level}\left(E_{j+1}\right)=i-1$, $i+\alpha-1=\operatorname{level}\left(E_{j}^{\prime}\right)=\operatorname{level}\left(E_{j+1}^{\prime}\right)$. According to Proposition $5, j$ is odd. Here there are three possible scenarios:

(2a) $i-1=\operatorname{level}\left(E_{j+2}\right), i+\alpha-1=\operatorname{level}\left(E_{j+2}^{\prime}\right)$.

(2b) $i=\operatorname{level}\left(E_{j+2}\right), i+\alpha=\operatorname{level}\left(E_{j+2}^{\prime}\right)$.

(2c) $i=\operatorname{level}\left(E_{j+2}\right), i+\alpha-1=\operatorname{level}\left(E_{j+2}^{\prime}\right)$.

The proof of these cases is analogous to that of cases (1a)-(1c) above.

Proof. [Proof of Theorem 3] Let $\Pi$ be an optimal type-2 possibility measure whose credal set has the maximum number of extreme points. This only happens if the level of any $E_{j}$ is the minimum possible, or, equivalently, if level $\left(E_{j}\right)=0$ for any $j=2, \ldots, 2 n$. It follows that the focal sets of $\Pi$ must be:

$$
\begin{aligned}
& E_{2 j+1}=A_{\left(x_{j}, y_{j}\right)}, \text { for any } j=1, \ldots, n-1, \\
& E_{2 j}=A_{\left(x_{j}, y_{j+1}\right)} \text { or } E_{2 j}=A_{\left(x_{j+1}, y_{j}\right)} \text { for any } j=1, \ldots, n-1, \\
& E_{2 n}=A_{\left(x_{n}, y_{m+1}\right)}, \ldots, E_{n+m-1}=A_{\left(x_{n}, y_{m}\right)},
\end{aligned}
$$

and also either $E_{1}=A_{\left(x_{1}, y_{1}\right)}$ or $E_{1}=\emptyset$.

If $\Pi^{\prime}$ is another type-2 optimal possibility different than $\Pi$, it must have some focal set $E_{i}$ with level $\left(E_{i}^{\prime}\right)>0$ for some $i$, and therefore from Proposition 7 the core of $\Pi$ has more extreme points than that of $\Pi^{\prime}$.

Consider the case $E_{1}=A_{\left(x_{1}, y_{1}\right)}$; applying Proposition 5 , the values $k_{j}$ associated with $\Pi$ are:

- $k_{1}=1$.

- For $j=1, \ldots, n-1, k_{2 j}=j$.

- For $j=2, \ldots, n, k_{2 j-1}=j$.

- For $j=2 n, \ldots, n+m-1, k_{j}=n$.

By Equation (4), the number of extreme points of $\mathcal{M}(\Pi)$ is:

$$
\begin{aligned}
k_{1} & \prod_{j=2}^{n+m-1}\left(1+k_{j}\right)=k_{1} \prod_{j=1}^{n-1}\left(1+k_{2 j}\right) \prod_{j=2}^{n}\left(1+k_{2 j-1}\right) \prod_{j=2 n}^{n+m-1}\left(1+k_{j}\right) \\
& =1 \prod_{j=1}^{n-1}(1+j) \prod_{j=2}^{n}(1+j) \prod_{j=2 n}^{n+m-1}(n+1) \\
& =(n !)\left(\frac{n !}{2}(n+1)\right)(n+1)^{m-n}=\frac{(n !)^{2}}{2}(n+1)^{m-n+1} .
\end{aligned}
$$

When $E_{1}=\emptyset$, we deduce from Proposition 2 that the maximal number of extreme points is the same as in the previous case.

Next we focus on the minimal number of extreme points. As we have seen in Proposition 7 , the greater the levels, the smaller the number of extreme points. We consider thus the following cases:

- If $j \leq m$, the maximum level for $E_{j}=A_{\left(x_{r}, y_{s}\right)}$ is achieved when either $r=j, s=1$ or $r=1, s=j$. Therefore, either $E_{j}=A_{\left(x_{j}, y_{1}\right)}$ or $E_{j}=A_{\left(x_{1}, y_{j}\right)}$. 
- If $j>m$, the maximum level for $E_{j}=A_{\left(x_{r}, y_{s}\right)}$ is achieved when either $r=m, s=j-m+1$ or $r=j-m+1, s=m$. Therefore, either $E_{j}=$ $A_{\left(x_{m}, y_{j-m+1}\right)}$ or $E_{j}=A_{\left(x_{j-m+1}, y_{m}\right)}$.

Since the focal sets must be nested, there are two possibilities:

(1) If $n<m$, then the focal sets must be:

$$
E_{j}=A_{\left(x_{1}, y_{j}\right)} \forall j=2, \ldots, m, \quad E_{j}=A_{\left(x_{j-m+1}, y_{m}\right)} \forall j=m+1, \ldots, m+n-1 .
$$

(2) If $n=m$, then the focal sets must be either as in Equation (13) or:

$$
E_{j}=A_{\left(x_{j}, y_{1}\right)} \forall j=2, \ldots, n, \quad E_{j}=A_{\left(x_{n}, y_{j-m+1}\right)} \forall j=m+1, \ldots, m+n-1 .
$$

According to Proposition 5, the values $k_{j}$ are given by:

$$
k_{j}= \begin{cases}1 & \text { for } j=1, \ldots, m . \\ m & \text { for } j=m+1, \ldots, m+n-1,\end{cases}
$$

and applying Equation (4), the number of extreme points is given by:

$$
k_{1}\left(1+k_{2}\right) \ldots\left(1+k_{n+m-1}\right)=2^{m-1}(m+1)^{n-1} .
$$

Lemma 3. Let $\Pi, \Pi^{\prime}$ be two optimal type-1 possibility measures. Assume that:

(1) The focal sets of $\Pi$ are given by $E_{1} \subset \ldots \subset E_{n+m-1}$, with $E_{1}=A_{\left(x_{n}, y_{m-1}\right)}^{c}$.

(2) The focal sets of $\Pi^{\prime}$ are given by $E_{1}^{\prime} \subset \ldots \subset E_{n+m-1}^{\prime}$, with $E_{1}^{\prime}=A_{\left(x_{n-1}, y_{m}\right)}^{c}$ and $E_{i}^{\prime}=E_{i}$ for any $i>1$.

Then, $|\operatorname{ext}(\mathcal{M}(\Pi))|=\left|\operatorname{ext}\left(\mathcal{M}\left(\Pi^{\prime}\right)\right)\right|$.

Proof. Let us denote by $k_{i}$ and $k_{i}^{\prime}$ the elements:

$$
k_{1}=\left|E_{1}\right|, \quad k_{i}=\left|E_{i} \backslash E_{i-1}\right|, \quad k_{1}^{\prime}=\left|E_{1}^{\prime}\right|, \quad k_{i}^{\prime}=\left|E_{i}^{\prime} \backslash E_{i-1}^{\prime}\right|,
$$

for any $i=2, \ldots, n+m-1$. Since $E_{i}=E_{i}^{\prime}$ for any $i>1, k_{i}=k_{i}^{\prime}$ for any $i=3, \ldots, n+m-1$. Let us compute the values of $k_{1}, k_{2}, k_{1}^{\prime}, k_{2}^{\prime}$. For this aim, note that since $E_{2}=E_{2}^{\prime}$, it must hold that $E_{2}=E_{2}^{\prime}=A_{\left(x_{n-1}, y_{m-1}\right)}^{c}$. Therefore:

$$
\begin{aligned}
k_{1} & =\left|E_{1}\right|=\left|A_{\left(x_{n}, y_{m-1}\right)}^{c}\right|=n m-n(m-1)=n . \\
k_{2} & =\left|E_{2} \backslash E_{1}\right|=\left|A_{\left(x_{n-1}, y_{m-1}\right)}^{c} \backslash A_{\left(x_{n}, y_{m-1}\right)}^{c}\right|=\left|A_{\left(x_{n}, y_{m-1}\right)}^{c} \backslash A_{\left(x_{n-1}, y_{m-1}\right)}\right| \\
& =n(m-1)-(n-1)(m-1)=m-1 . \\
k_{1}^{\prime} & =\left|E_{1}^{\prime}\right|=\left|A_{\left(x_{n-1}, y_{m}\right)}^{c}\right|=n m-(n-1) m=m . \\
k_{2}^{\prime} & =\left|E_{2}^{\prime} \backslash E_{1}^{\prime}\right|=\left|A_{\left(x_{n-1}, y_{m-1}\right)}^{c} \backslash A_{\left(x_{n-1}, y_{m}\right)}^{c}\right|=\left|A_{\left(x_{n-1}, y_{m}\right)}^{c} \backslash A_{\left(x_{n-1}, y_{m-1}\right)}\right| \\
& =(n-1) m-(n-1)(m-1)=n-1 .
\end{aligned}
$$

Then,

$$
k_{1}\left(1+k_{2}\right)=n m=k_{1}^{\prime}\left(1+k_{2}^{\prime}\right),
$$


whence

$$
\begin{aligned}
k_{1} & \prod_{i=2}^{n+m-1}\left(1+k_{i}\right)=k_{1}\left(1+k_{2}\right) \prod_{i=3}^{n+m-1}\left(1+k_{i}\right)=n m \prod_{i=3}^{n+m-1}\left(1+k_{i}\right) \\
= & n m \prod_{i=3}^{n+m-1}\left(1+k_{i}^{\prime}\right)=k_{1}^{\prime}\left(1+k_{2}^{\prime}\right) \prod_{i=3}^{n+m-1}\left(1+k_{i}^{\prime}\right)=k_{1}^{\prime} \prod_{i=2}^{n+m-1}\left(1+k_{i}^{\prime}\right),
\end{aligned}
$$

and therefore $\mathcal{M}(\Pi)$ and $\mathcal{M}\left(\Pi^{\prime}\right)$ have the same number of extreme points.

Lemma 4. Let $\Pi, \Pi^{\prime}$ be two optimal type-1 possibility measures. Assume that they satisfy the following properties:

(1) The focal sets of $\Pi$ are given by $E_{1} \subset \ldots \subset E_{n+m-1}$ such that $E_{i}=A_{\left(x_{r}, y_{s}\right)}^{c}$, $E_{i+1}=A_{\left(x_{r-1}, y_{s}\right)}^{c}$ and $E_{i+2}=A_{\left(x_{r-1}, y_{s-1}\right)}^{c}$.

(2) The focal sets of $\Pi^{\prime}$ are given by

$$
E_{1} \subset \ldots \subset E_{i} \subset E_{i+1}^{\prime} \subset E_{i+2} \subset \ldots \subset E_{n+m-1}
$$

(3) $s>r$

such that $E_{i}=A_{\left(x_{r}, y_{s}\right)}^{c}, E_{i+1}=A_{\left(x_{r}, y_{s-1}\right)}^{c}$ and $E_{i+2}=A_{\left(x_{r-1}, y_{s-1}\right)}^{c}$.

Then, $\left|\operatorname{ext}\left(\mathcal{M}\left(\Pi^{\prime}\right)\right)\right|>|\operatorname{ext}(\mathcal{M}(\Pi))|$.

Proof. From Equation (4), we only need to show that $\left(1+k_{i+1}\right)\left(1+k_{i+2}\right)<$ $\left(1+k_{i+1}^{\prime}\right)\left(1+k_{i+2}^{\prime}\right)$.

For $\Pi$, it holds that:

$$
\begin{aligned}
k_{i+1} & =\left|E_{i+1} \backslash E_{i}\right|=\left|A_{\left(x_{r-1}, y_{s}\right)}^{c} \backslash A_{\left(x_{r}, y_{s}\right)}^{c}\right|=\left|A_{\left(x_{r}, y_{s}\right)} \backslash A_{\left(x_{r-1}, y_{s}\right)}\right| \\
& =r s-(r-1) s=s \\
k_{i+2} & =\left|E_{i+2} \backslash E_{i+1}\right|=\left|A_{\left(x_{r-1}, y_{s-1}\right)}^{c} \backslash A_{\left(x_{r-1}, y_{s}\right)}^{c}\right|=\left|A_{\left(x_{r-1}, y_{s}\right)} \backslash A_{\left(x_{r-1}, y_{s-1}\right)}\right| \\
& =(r-1) s-(r-1)(s-1)=r-1 .
\end{aligned}
$$

On the other hand, since for any $j \neq i+1, i+2, k_{i+1}^{\prime}=\left|E_{i+1}^{\prime} \backslash E_{i}\right|$ and $k_{i+2}^{\prime}=$ $\left|E_{i+2} \backslash E_{i+1}^{\prime}\right|$, then $k_{j}^{\prime}=k_{j}$, and furthermore:

$$
\begin{aligned}
k_{i+1}^{\prime} & =\left|E_{i+1}^{\prime} \backslash E_{i}^{\prime}\right|=\left|A_{\left(x_{r}, y_{s-1}\right)}^{c} \backslash A_{\left(x_{r}, y_{s}\right)}^{c}\right|=\left|A_{\left(x_{r}, y_{s}\right)} \backslash A_{\left(x_{r}, y_{s-1}\right)}\right| \\
& =r s-r(s-1)=r . \\
k_{i+2}^{\prime} & =\left|E_{i+2}^{\prime} \backslash E_{i+1}^{\prime}\right|=\left|A_{\left(x_{r-1}, y_{s-1}\right)}^{c} \backslash A_{\left(x_{r}, y_{s-1}\right)}^{c}\right|=\left|A_{\left(x_{r}, y_{s-1}\right)} \backslash A_{\left(x_{r-1}, y_{s-1}\right)}\right| \\
& =r(s-1)-(r-1)(s-1)=s-1 .
\end{aligned}
$$

Since $s>r$,

$$
\left(1+k_{i+1}\right)\left(1+k_{i+2}\right)=(s+1) r<(r+1) s=\left(1+k_{i+1}^{\prime}\right)\left(1+k_{i+2}^{\prime}\right) .
$$

Consequently, $\mathcal{M}\left(\Pi^{\prime}\right)$ has more extreme points than $\mathcal{M}(\Pi)$. 
Lemma 5. Let $\Pi$ and $\Pi^{\prime}$ be two optimal type-1 possibility measures on $\mathcal{P}(\mathcal{X} \times \mathcal{Y})$, with respective focal sets $E_{1} \subset \ldots \subset E_{n+m-1}$ and $E_{1}^{\prime} \subset \ldots \subset E_{n+m-1}^{\prime}$. Assume that level $\left(E_{j}^{\prime}\right)-\operatorname{level}\left(E_{j}\right) \in\{0,1\}$ for any $j=2, \ldots, n+m-1$ and that level $\left(E_{j}^{\prime}\right)-$ level $\left(E_{j}\right)=1$ for at least one $j$. Then, it holds that:

(1) If level $\left(E_{j}^{\prime}\right)-\operatorname{level}\left(E_{j}\right)=1$ only for $j=1,|\operatorname{ext}(\mathcal{M}(\Pi))|=\left|\operatorname{ext}\left(\mathcal{M}\left(\Pi^{\prime}\right)\right)\right|$.

(2) Otherwise, $|\operatorname{ext}(\mathcal{M}(\Pi))|>\left|\operatorname{ext}\left(\mathcal{M}\left(\Pi^{\prime}\right)\right)\right|$.

Proof. The first case follows from Lemma 3, taking into account that two focal sets coincide when their levels coincide.

The proof of the second statement is analogous to that of Lemma 2 by using Lemma 4.

Proof. [Proof of Proposition 8] When the inequality level $\left(E_{j}\right) \leq \operatorname{level}\left(E_{j}^{\prime}\right)$ is strict only for $j=1$, we are in the conditions of Lemma 3 , so both $\mathcal{M}(\Pi)$ and $\mathcal{M}\left(\Pi^{\prime}\right)$ have the same number of extreme points.

For the other case, the proof is analogous to that of Proposition 7 by using Lemmas 3 and 5 .

Proof. [Proof of Theorem 4] In order to obtain the maximum number of extreme points, the focal sets of $\Pi$ must have the minimum possible level. Let $\widetilde{\Pi}$ be the optimal type- 2 possibility measure associated with $\Pi$. From Theorem 3 we know that the focal sets of $\widetilde{\Pi}, \widetilde{E}_{1}, \ldots, \widetilde{E}_{n+m-1}$, have the minimum possible level when they are given by:

$$
\begin{aligned}
& \widetilde{E}_{2 j+1}=A_{\left(x_{j}, y_{j}\right)}, \text { for any } j=0, \ldots, n-1 . \\
& \widetilde{E}_{2 j}=A_{\left(x_{j}, y_{j+1}\right)} \text { or } \widetilde{E}_{2 j}=A_{\left(x_{j+1}, y_{j}\right)}, \text { for any } j=1, \ldots, n-1 . \\
& \widetilde{E}_{2 n}=A_{\left(x_{n}, y_{m+1}\right)}, \ldots, \widetilde{E}_{n+m-1}=A_{\left(x_{n}, y_{m}\right)} .
\end{aligned}
$$

Then, the focal sets of $\Pi$ must be:

$$
\begin{aligned}
& E_{j}=\widetilde{E}_{m+n-j-1}^{c}=A_{\left(x_{n}, y_{m-j}\right)}^{c}, \text { for any } j=1, \ldots, m-n-1 . \\
& E_{n+m-2 j}=\widetilde{E}_{2 j-1}^{c}=A_{\left(x_{j}, y_{j}\right)}^{c} \text { for any } j=1, \ldots, n . \\
& E_{n+m-2 j-1}=\widetilde{E}_{2 j}^{c}=A_{\left(x_{j}, y_{j+1}\right)}^{c} \text { or } E_{n+m-2 j-1}=\widetilde{E}_{2 j}^{c}=A_{\left(x_{j+1}, y_{j}\right)}^{c} \\
& \quad \quad \text { for any } j=1, \ldots, n-1 . \\
& E_{m+n-1}=\mathcal{X} \times \mathcal{Y} .
\end{aligned}
$$

Furthermore, since $k_{i}=\widetilde{k}_{m+n-j}$, and therefore, making use of Theorem 3:

$$
\begin{aligned}
& k_{j}=\widetilde{k}_{m+n-j}=n, \text { for any } j=1, \ldots, m-n-1 . \\
& k_{n+m-2 j}=\widetilde{k}_{2 j}=j, \text { for any } j=1, \ldots, n . \\
& k_{n+m-2 j-1}=\widetilde{k}_{2 j+1}=j+1, \text { for any } j=1, \ldots, n-1 . \\
& k_{m+n-1}=1 .
\end{aligned}
$$


This means that the maximum number of extreme points is:

$$
\begin{aligned}
k_{1} & \prod_{j=1}^{n+m-1}\left(1+k_{j}\right) \\
= & n \prod_{j=2}^{m-n-1}\left(1+k_{j}\right) \prod_{j=1}^{n}\left(1+k_{n+m-2 j}\right) \prod_{j=1}^{n-1}\left(1+k_{n+m-2 j-1}\right)\left(1+k_{m+n-1}\right) \\
= & 2 n \prod_{j=2}^{m-n-1}(n+1) \prod_{j=1}^{n}(j+1) \prod_{j=1}^{n-1}(j+2) \\
= & 2 n(n+1)^{m-n-2}(n+1) ! \frac{(n+1) !}{2}=n(n+1)^{m-n}(n !)^{2} .
\end{aligned}
$$

Consider now the case $m=n+1$. Then, as we have seen, the following focal sets determine an optimal type-1 possibility $\Pi$ with the maximum number of extreme points:

$$
\begin{aligned}
& E_{2 j-1}=A_{\left(x_{n-j+1}, y_{n-j+1}\right)}^{c}, \text { for any } j=1, \ldots, n . \\
& E_{2 j}=A_{\left(x_{n-j+1}, y_{n-j}\right)}^{c} \text { or } E_{2 j}=A_{\left(x_{n-j}, y_{n-j+1}\right)}^{c}, \text { for any } j=1, \ldots, n .
\end{aligned}
$$

If we replace the focal set $E_{1}=A_{\left(x_{n}, y_{n}\right)}^{c}$ by $E_{1}^{\prime}=A_{\left(x_{n-1}, y_{n+1}\right)}^{c}$, then by Lemma 3 we obtain an optimal type-1 possibility inducing the same number of extreme points than $\Pi$.

With respect to the minimal number of extreme points, it follows from Proposition 8 that they are attained when the levels of the focal sets are as large as possible. Taking into account the correspondence with an optimal type-2 possibility measure in Equation (11) and Equation (14), we obtain

$$
\widetilde{k}_{j}= \begin{cases}1 & \text { for } j=1, \ldots, m \\ m & \text { for } j=m+1, \ldots, n+m-1,\end{cases}
$$

and then Equation (12) means that the associated number of extreme points is $m(1+m)^{n-2} 2^{m}$. 